ARTICLE

\title{
Immunological imprinting of the antibody response in COVID-19 patients
}

\author{
Teresa Aydillo (1) 1,2, Alexander Rombauts (10) 3,4, Daniel Stadlbauer (10) 1, Sadaf Aslam 1,2, \\ Gabriela Abelenda-Alonso 3,4, Alba Escalera1,2,5, Fatima Amanat1,5, Kaijun Jiang1, Florian Krammer (10 1,6凶, \\ Jordi Carratala (i) ${ }^{3,4 \times}$ \& Adolfo García-Sastre (iD $1,2,7,8 \times$
}

In addition to severe acute respiratory syndrome coronavirus 2 (SARS-CoV-2), humans are also susceptible to six other coronaviruses, for which consecutive exposures to antigenically related and divergent seasonal coronaviruses are frequent. Despite the prevalence of COVID19 pandemic and ongoing research, the nature of the antibody response against severe acute respiratory syndrome coronavirus 2 (SARS-CoV-2) is unclear. Here we longitudinally profile the early humoral immune response against SARS-CoV-2 in hospitalized coronavirus disease 2019 (COVID-19) patients and quantify levels of pre-existing immunity to OC43, HKU1 and $229 E$ seasonal coronaviruses, and find a strong back-boosting effect to conserved but not variable regions of OC43 and HKU1 betacoronaviruses spike protein. However, such antibody memory boost to human coronaviruses negatively correlates with the induction of $\lg G$ and IgM against SARS-CoV-2 spike and nucleocapsid protein. Our findings thus provide evidence of immunological imprinting by previous seasonal coronavirus infections that can potentially modulate the antibody profile to SARS-CoV-2 infection.

\footnotetext{
${ }^{1}$ Department of Microbiology, Icahn School of Medicine at Mount Sinai, New York, NY, USA. ${ }^{2}$ Global Health and Emerging Pathogens Institute, Icahn School of Medicine at Mount Sinai, New York, NY, USA. ${ }^{3}$ Department of Infectious Diseases, Bellvitge University Hospital, Bellvitge Biomedical Research Institute (IDIBELL), University of Barcelona, L'Hospitalet de Llobregat, Barcelona, Spain. ${ }^{4}$ Spanish Network for Research on Infectious Diseases (REIPI, RD16/0016, Carlos III Health Institute, Madrid, Spain. ${ }^{5}$ Graduate School of Biomedical Sciences, Icahn School of Medicine at Mount Sinai, New York, NY, USA.

${ }^{6}$ Department of Pathology, Icahn School of Medicine at Mount Sinai, New York, NY, USA. ${ }^{7}$ Division of Infectious Disease, Department of Medicine, Icahn School of Medicine at Mount Sinai, New York, NY, USA. ${ }^{8}$ The Tisch Cancer Institute, Icahn School of Medicine at Mount Sinai, New York, NY, USA.

凶email: florian.krammer@mssm.edu; jcarratala@bellvitgehospital.cat; adolfo.garcia-sastre@mssm.edu
} 
S ince January 2020, the Severe Acute Respiratory Syndrome Coronavirus 2 (SARS-CoV-2) virus has been spreading globally causing the first documented pandemic of coronavirus in history ${ }^{1,2}$. SARS-CoV-2 is a betacoronavirus that belongs to a large family of viruses capable to infect both mammals and birds. Humans are susceptible to at least other six viruses from the genus alpha and betacoronavirus ${ }^{3}$. All of them typically cause respiratory illness but to a different extent. While SARS-CoV-1 and Middle East Respiratory Syndrome Coronavirus, are highly pathogenic betacoronaviruses that have caused zoonotic outbreaks in humans in the last 20 years $^{4,5}$, the alphacoronaviruses $229 \mathrm{E}$ and NL63, and the betacoronaviruses OC43 and HKU1, frequently cause mild upper respiratory tract disease and have been circulating in humans as seasonal viruses $^{3,6}$. The ongoing pandemic of coronavirus disease 2019 (COVID-19), the disease caused by SARS-CoV-2, is still challenging healthcare systems and the research community. SARSCoV-2 can cause a different range of clinical manifestations, from asymptomatic to severe respiratory syndrome. However, a high percentage of severe cases have been reported and estimated numbers of patients that succumbed to COVID-19 disease are more than 3 million according to WHO as May 20212,7 (https:// covid19.who.int/). Many vaccine candidates are being tested in clinical trials and several have already been authorized for use in the population ${ }^{8-10}$. However, we are still in an early phase and studies regarding vaccine effectiveness in special populations are needed. Similarly, longevity of the humoral immunity after infection and vaccination is still an ongoing debate.

One of the main targets of antibody responses to coronaviruses is the spike, the surface glycoprotein that mediates attachment to the host receptor and membrane fusion. Two subunits can be identified, the S1 subunit containing the receptor-binding domain (RBD), essential for binding to the entry receptor ${ }^{11-13}$; and the S2 subunit, responsible of virus cell fusion ${ }^{14}$. Different human coronaviruses use different domains to bind their human receptors and to mediate cell entry. While the human endemic betacoronaviruses OC43 and HKU1, bind to sialic acids, 229E alphacoronavirus uses human aminopeptidase $\mathrm{N}$ as a cellular determinant for susceptibility ${ }^{15,16}$. NL63, SARS-CoV, and SARS$\mathrm{CoV}-2$, in contrast, need direct interaction with the angiotensinconverting enzyme 2 to infect cells ${ }^{13,17}$. Therefore, antibodies directed against the RBD of human coronaviruses are capable to neutralize the virus ${ }^{15,18,19}$ and no cross-reactive neutralizing antibodies among seasonal human coronavirus are expected due to the high specificity of this process and the sequence divergence between the RBD of these viruses ${ }^{20-23}$. In addition, the more cross-reactive viral nucleoprotein $(\mathrm{N})$ has also shown to be immunogenic and induce antibodies in COVID-19 patients. However, in contrast to RBD antibodies, $\mathrm{N}$ antibodies are not able to neutralize the virus in tissue culture ${ }^{11,23,24}$.

Several studies have demonstrated that $\mathrm{T}$ cells can recognize homologous epitopes shared between different endemic coronaviruses ${ }^{25-30}$. However, serum cross-reactivity between conserved epitopes from SARS-CoV-2 and seasonal human coronaviruses is still under investigation ${ }^{23,31-33}$ and the role of preexisting humoral immunity and immunodominance for B cell responses needs to be addressed. Immune imprinting (or original antigenic sin), refers to the preference of the immune system to recall existing memory cells, rather than stimulating de novo responses when encountering a novel but closely related antigen $^{34}$. This has been shown for viruses like influenza virus, in which subsequent infections with antigenically related strains produce a recall response or 'back-boosting' that generates an increase in antibody titers toward epitopes shared between the current and the historic strains encountered earlier in life ${ }^{35-38}$. Boost of cross-reactive antibody responses can also occur for viruses like dengue virus (DENV) upon secondary infections with a different serotype $\mathrm{e}^{39,40}$. In this case, specific titers to the original DENV were higher than those specific to the second infecting DENV upon secondary DENV infection ${ }^{41,42}$.

Here, we profile the antibody responses of a longitudinal cohort of hospitalized patients with COVID-19. We characterize de novo antibody responses against SARS-CoV-2 and preexisting immunity against selected endemic coronavirus being targeted by the humoral immune system to investigate the role of immunological imprinting on COVID-19 patients' antibody response. We show that the induction of antibodies against conserved epitopes of seasonal coronaviruses may hinder the induction of specific antibodies toward divergent SARS-CoV-2 antigens. This study provides a dynamic characterization of the co-evolving nature of antibody responses to human coronaviruses, both seasonal and pandemic, and contributes to a better understanding of cross-reactive antibody responses and $\mathrm{B}$ cells immunodominance against human coronaviruses.

\section{Results}

The BACO cohort. Thirty-seven COVID-19 patients were recruited at the University Hospital of Bellvitge during the first wave of SARS-CoV-2 in Barcelona (Spain) from March 26, 2020 to May 28, 2020. Mean age was 65 years and $67 \%$ were male. Chronic comorbidities were frequent among COVID-19 patients $(25,67.7 \%)$. In particular, 16 (43.2\%) of patients were obese (body mass index $>30$ ) at the time of hospitalization. A high percentage of patients had respiratory symptoms, such as coughing (26, $70.3 \%)$ and dyspnea $(14,37.8 \%)$, whereas diarrhea was also present in seven (18.9\%) of the patients. While no remdesivir was available, lopinavir/ritonavir was used for 17 (45.9\%) patients. All patients, except one $(36,97.3 \%)$, developed SARS-CoV-2 viral pneumonia and four $(10.8 \%)$ required intensive care unit admission. Five (13.5\%) patients died. Demographics, clinical characteristics, interventions, such as drug therapy and outcomes are detailed in Table 1.

Acute blood samples were collected longitudinally in the BACO cohort at the recruitment upon hospital admission, and at days 3 and 7 in $33(89.1 \%)$ and 22 (59.4\%) patients, respectively. Mean time from symptom onset to inclusion in the study was 7 days (range 2-14). Most of the patients $(25,67.5 \%)$ were recruited within the first week of symptom onset, whereas 12 (32.4\%) patients had longer periods until hospitalization. COVID-19 survivors were followed up in the convalescence period and 28 out of 32 survivors $(87.5 \%)$ had another blood draw after hospital discharge with a mean time of 46 days post recruitment (range, 30-56 days).

COVID-19 patients developed anti-SARS-CoV-2 antibodies linked to back-boosting of antibodies against S2 domain of betacoronaviruses. To profile the early antibody response in COVID-19 patients, we investigated the levels of neutralizing antibodies against authentic SARS-CoV-2 virus and IgG/IgM ELISAs against multiple antigens including the full-length spike (S), the spike RBD $\mathrm{S}$ and the $\mathrm{N}$ of SARS-CoV-2. IgG and IgM levels were quantified as area under the curve (AUC) by plotting normalized optical density (OD) values against the reciprocal serum sample dilutions for ELISAs (Supplementary Fig. 1A). To improve visualization, the longitudinal antibody profile of each individual patient together with the geometric mean titer (GMT, CI 95\%) at each time point is shown for AUC ELISA and neutralizing titers in Fig. $1 \mathrm{~A}$ and Supplementary Table 1. All patients developed detectable levels of neutralizing antibodies at day 7 post recruitment while levels remained stable during the convalescent phase, except for two survivors. Similar responses were 


\section{Table 1 Demographics and clinical characteristics of the BACO cohort.}

Total $(n=37)$

\begin{tabular}{|c|c|}
\hline \multicolumn{2}{|l|}{ Demographics and comorbidities } \\
\hline Age (mean, IQR) & $67(25)$ \\
\hline $\operatorname{Men}(n, \%)$ & $25(67.6)$ \\
\hline Comorbidities $(n, \%)$ & $25(67.7)$ \\
\hline Lung disease $(n, \%)$ & $7(18.9)$ \\
\hline Diabetes mellitus & $7(18.9)$ \\
\hline Heart disease $(n, \%)$ & $5(13.5)$ \\
\hline Kidney disease $(n, \%)$ & $3(8.1)$ \\
\hline Obesity $(n, \%)$ & $16(43.2)$ \\
\hline $\operatorname{SOTR}(n, \%)$ & $1(2.7)$ \\
\hline \multicolumn{2}{|l|}{ Signs and symptoms } \\
\hline $\begin{array}{l}\text { Days from symptom onset to enrollment } \\
\text { (mean, range) }\end{array}$ & $7.19(2-14)$ \\
\hline Days of fever (mean, range) & $4.68(0-12)$ \\
\hline Throat ache $(n, \%)$ & $4(10.8)$ \\
\hline Cough $(n, \%)$ & $26(70.3)$ \\
\hline Dyspnea $(n, \%)$ & $14(37.8)$ \\
\hline Diarrhea $(n, \%)$ & $7(18.9)$ \\
\hline $\mathrm{Sp02}<94 \%(n, \%)$ & $14(37.8)$ \\
\hline \multicolumn{2}{|l|}{ Drug therapy } \\
\hline Hydroxychloroquine $(n, \%)$ & $36(97.3)$ \\
\hline Lopinavir/Ritonavir $(n, \%)$ & $17(45.9)$ \\
\hline Tocilizumab $(n, \%)$ & $10(27)$ \\
\hline Antibiotics $(n, \%)$ & $19(51.4)$ \\
\hline Corticosteroids $(n, \%)$ & $18(48.6)$ \\
\hline \multicolumn{2}{|l|}{ Outcomes } \\
\hline Pneumonia $(n, \%)$ & $36(97.3)$ \\
\hline $\operatorname{ICU}(n, \%)$ & $4(10.8)$ \\
\hline Days from hospitalization to ICU (mean, range) & $9.5(5-12)$ \\
\hline Days in ICU (mean, range) & $15(15-22)$ \\
\hline Non-mechanical ventilation $(n, \%)$ & $11(29.7)$ \\
\hline Mechanical ventilation $(n, \%)$ & $2(5.4)$ \\
\hline Nosocomial co-infection $(n, \%)$ & $2(5.4)$ \\
\hline Mortality $(n, \%)$ & $5(13.5)$ \\
\hline Days of hospitalization (mean, range) & $11.2(2-47)$ \\
\hline
\end{tabular}

SOTR solid organ transplant recipient, $\mathrm{SpO} 2<94 \%$ pulse oximetry below $94 \%, I C U$ intensive care unit.

found by ELISA, although higher levels of antibodies against IgG $\mathrm{S}$ compared to IgG RBD were present. When comparing to the induction of anti-spike antibodies, the IgG isotype reached higher titers than the IgM isotype, whereas anti-N protein IgG had similar induction than the anti-S IgG. We then determined fold increase of antibody titers from baseline levels. Overall, all patients had a high induction of SARS-CoV-2 S and RBD antibodies at day 7 post recruitment. IgG titers against the $S$ and $R B D$ of SARS-CoV-2 remained stable at the convalescent time point with similar levels compared to peak titers at day 7 . By contrast, IgM against the $\mathrm{S}, \operatorname{IgG}$ against $\mathrm{N}$ and neutralizing titers against authentic SARS-CoV-2 virus decreased to levels resembling those at day 3. Geometric mean fold rise (GMFR) and adjusted $p$ values on pairwise comparisons after related samples Friedman's twoway ANOVA at each time points are shown Fig. 1B. We next tested the correlation between neutralization activity and levels of anti-SARS-CoV-2 antibodies. Scatterplot matrices shown in Supplementary Fig. 2 indicate that the antibodies detected against SARS-CoV-2 antigens correlated well with neutralizing activity, with Pearson $\mathrm{R}^{2}$ ranging from 69 to $81 \%$ in the case of IgG against the RBD $S$ of SARS-CoV-2.

The $S$ gene of SARS-CoV-2 is highly divergent from human seasonal coronaviruses (hCoV). Infection with endemic $\mathrm{hCoV}$ in humans happens frequently $3,6,43$, causing mild respiratory disease. Multiple sequence alignment (MSA) between the $\mathrm{S}$ of
SARS-CoV-2 and selected seasonal coronaviruses showed amino acid identity ranging from $28 \%$ for alphacoronaviruses (229E) and $32.5 \%$ and $33 \%$ for betacoronaviruses (OC43 and HKU1, respectively). To identify conserved amino acid regions, we also estimated the relative conservation scores of the $S$ protein of SARS-CoV-2 using the chain A of the SARS-CoV-2 spike protein in the closed state as a reference. MSA and relative amino acid conservation was determined by using the ConSurf server. Figure $2 \mathrm{~A}$ shows the conservation score for each amino acid position and projected on the S protein structure. Evolutionary conservation analysis showed that the S2 subunit had the highest degree of identity among the sequences tested. Given the high probability of previous exposure to seasonal coronaviruses in the BACO cohort, we screened levels of antibodies against the spike of alphacoronavirus 229E and betacoronaviruses HKU1, OC43. Antigens tested included full-length $S$ protein for all three endemic coronaviruses together with the less conserved HKU1 S1 subunit (Supplementary Fig. 3A). Remarkably, COVID-19 patients exhibited an outstanding back-boosting of antibodies to the beta- $\mathrm{CoV}$ spikes tested, with similar a longitudinal profile as the one observed for the SARS-CoV-2 spike and for SARS-CoV-2 neutralizing titers (Fig. 3A). The back-boost was higher at day 7 , with a GMFR from baseline levels of 3.8 and 4 for HKU1 S and OC43 S, respectively (Fig. 3B, Supplementary Table 1). While IgG levels against 229E were already high at baseline, no increase was detected at any time point during the follow-up on patients with COVID-19. Interesting, no back-boosting was found when we tested antibody titers against the more divergent $\mathrm{S} 1$ subunit of HKU1, pointing to an increase of immune responses towards conserved epitopes of the S2 subunit of the spike protein of betahuman coronaviruses. Similar to influenza viruses, HKU1 and OC43 use sialic acids as canonical receptor to infect human cells ${ }^{16}$. This is mediated by an additional surface protein in these viruses with hemagglutination (HA) activity (hemagglutininesterase (HE) protein). No increase in OC43 HA inhibitory antibodies was found in COVID-19 patients, consistent with the lack of HE in SARS-CoV-2. Longitudinal profile and fold increase antibody titers to selected seasonal human coronaviruses antigens are shown in Fig. 3 and Supplementary Table 1.

To test whether the antibody response characterized in the BACO cohort correlated with disease trajectory, we grouped patients according to disease phenotype. Patients were assigned as mild/moderate $(N=26,70.3 \%)$ or severe/severe end-of organ disease (EOD, $N=11,29.7 \%$ ) based on a previously described severity scale ${ }^{44}$. No statistically significant differences were found between humoral immune response in patients with mild and severe/severe EOD disease, but the latter tended to have a delay in the antibody response towards SARS-CoV-2 antigens compared to moderate cases (Fig. 4A). Patients with severe disease had lower Ct values, and therefore higher viral loads (Fig. 4B). Besides, a positive correlation was found between anti- SARS-CoV2 antibodies and mean $\mathrm{Ct}$ values in paired nasopharyngeal swabs of COVID-19 patients acknowledging an interplay between antibodies and virus control and disease severity in COVID-19 patients. However, no correlation was found between antibodies against seasonal coronaviruses and viral loads in the BACO Cohort (Fig. 4C).

Immunological imprinting results in a bias in the induction of antibodies to conserved vs. variable regions of the SARS-COV2 spike. Given the strong back-boosting observed to the conserved epitopes of the $S$ domains of human betacoronaviruses in patients with COVID-19, we next investigated whether a strong back-boosting might reduce the induction of de novo humoral immune responses against specific epitopes of the spike of SARS$\mathrm{CoV}-2$ defined as fold induction over baseline levels. 

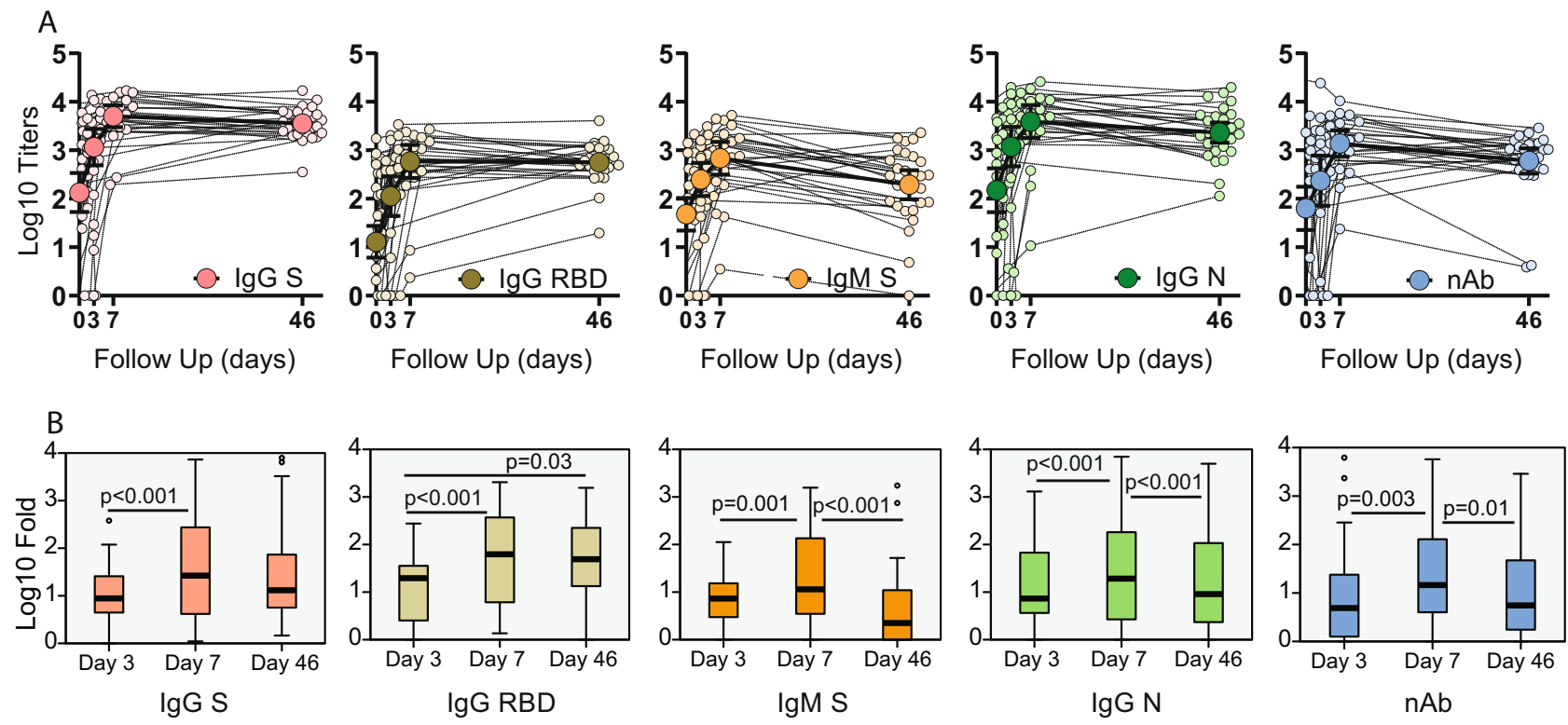

Fig. 1 Longitudinal antibody response to SARS-CoV-2 antigens. Serum from hospitalized COVID-19 patients was analyzed at baseline, at hospital recruitment and days 3 and 7. A subsequent sample was collected in the convalescence period in the COVID-19 survivors with mean time of 46 days. A Longitudinal profile of antibodies against SARS-CoV-2. Antibody titer was quantified as area under the curve (AUC) after serial serum dilution for each sample (Supplementary Fig. 1). Calculated AUC at each time is shown to quantify changes over time for each individual (small dots) against immunoglobulin G (IgG) spike, IgG receptor-binding domain (RBD), immunoglobulin M (IgM) spike and IgG nucleocapsid (N); and neutralizing activity (nAb) as inhibitory concentration 50\% (IC50\%). Geometric mean titer (GMT, big dots) and confidence interval (Cl 95\%) are also shown. B Boxplot diagram of geometric mean fold rise (GMFR) antibody titers against SARS-CoV-2 at the same time points: IgG spike, IgG RBD, IgM spike and IgG NP; and neutralizing activity $(n A b)$. Related-samples Friedman's two-way ANOVA was performed. Significant adjusted $p$ values after pairwise comparisons are shown for each comparison. Black bar indicates GMFR values, box indicates IQR (Q1-Q3), lines indicate minimum and maximum. Outliers from the observed distribution are shown. Total $n=116$ biologically independent serum samples (day $0=37$, day $3=29$, day $7=22$, day $46=28$ ). $n=116$ biological samples examined against four different SARS-CoV-2 substrates for ELISA assays; ELISAs for each substrate were run once each. $N=116$ serum samples examined over two independent experiments for neutralization assays.

A
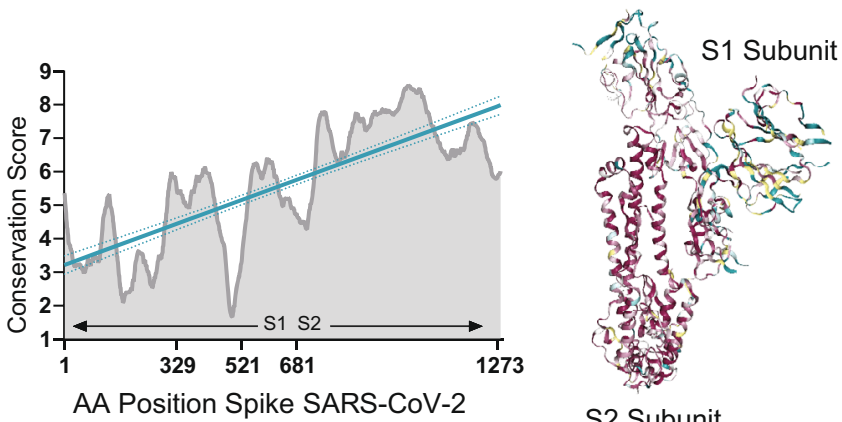

S2 Subunit

Fig. 2 Conservation of SARS-CoV-2 S protein. A Multiple sequence alignment was generated by the ConSurf algorithm (https://consurf.tau.ac.il) using the chain A of the SARS-CoV-2 spike protein in the closed state (PDB ID $6 \mathrm{VXX}$ ) as a reference. Amino acid conservation scores were classified into nine levels. Structure of the SARS-CoV-2 S protein (chain A) with amino acid residues colored according to conservation on a scale from green (1, most variable) to dark purple $(9$, most conserved) is also shown.

To test this hypothesis, we examined the relationship between pre-exposure to HKU1, OC43, and 229E viruses and the induction of SARS-CoV-2 S, RBD, and $\mathrm{N}$ antibodies in our cohort, and determined Pearson correlation coefficients between IgG levels at baseline against seasonal human coronaviruses and the fold induction of SARS-CoV-2 antigens at days 3, 7 and convalescence. Pearson correlation matrices according to seasonal coronavirus subtype are shown in Fig. 5. Striking differences were found according to virus types. While pre-existing IgG levels against HKU1 and OC43 spike protein negatively impacted the induction of de novo IgG and IgM against SARS-CoV-2 antigens, including $\mathrm{S}$ and $\mathrm{N}$ protein (Fig. $5 \mathrm{~A}, \mathrm{~B}$ ), no influence was found when testing the relationship between pre-existing anti-229E spike IgG levels (Fig. 5D). Moreover, correlations became stronger over time, and while this correlation was lower at day 3 , a stronger correlation was found at day 7 , and convalescence time points in the surviving patients. Besides, a comparable performance was observed when testing the subsequent induction of the IgG antibodies against the variable RBD domain of SARSCoV-2 spike. This result suggests that pre-existing immunity against seasonal betacoronaviruses biases the humoral response towards betacoronaviruses cross-reactive antibodies in detriment of antibodies against the more divergent and antigenically unique domains of the $\mathrm{S}$ of SARS-CoV-2, such as those of the RBD domain (Fig. 5A, B). This was also evidenced by the lack of impact of pre-existing HKU1 S1 IgG levels (S1 is divergent and harbors the RBD) on specific SARS-CoV-2 antibodies induction (Fig. 5C). Thus, only the levels of antibodies against cross-reactive epitopes of human betacoronaviruses had an effect on the subsequent antibody response to SARS-CoV-2 unique spike antigens. Because neutralization activity has been linked to in vivo protection after challenge with SARS-CoV- $2^{45}$, we also tested if immune imprinting could hinder the induction of neutralizing antibodies against SARS-CoV-2. No significant correlation was found. However, linear regression analysis determined a standardized beta coefficient of $-0.32(95 \%$ CI $-0.35-0.05, p=0.13)$ and $-0.31(95 \%$ CI $-0.28-0.02, p=0.1)$ at day 7 and convalescence time points, respectively, for pre-existing HKU1 spike antibody levels approximating a negative impact of 

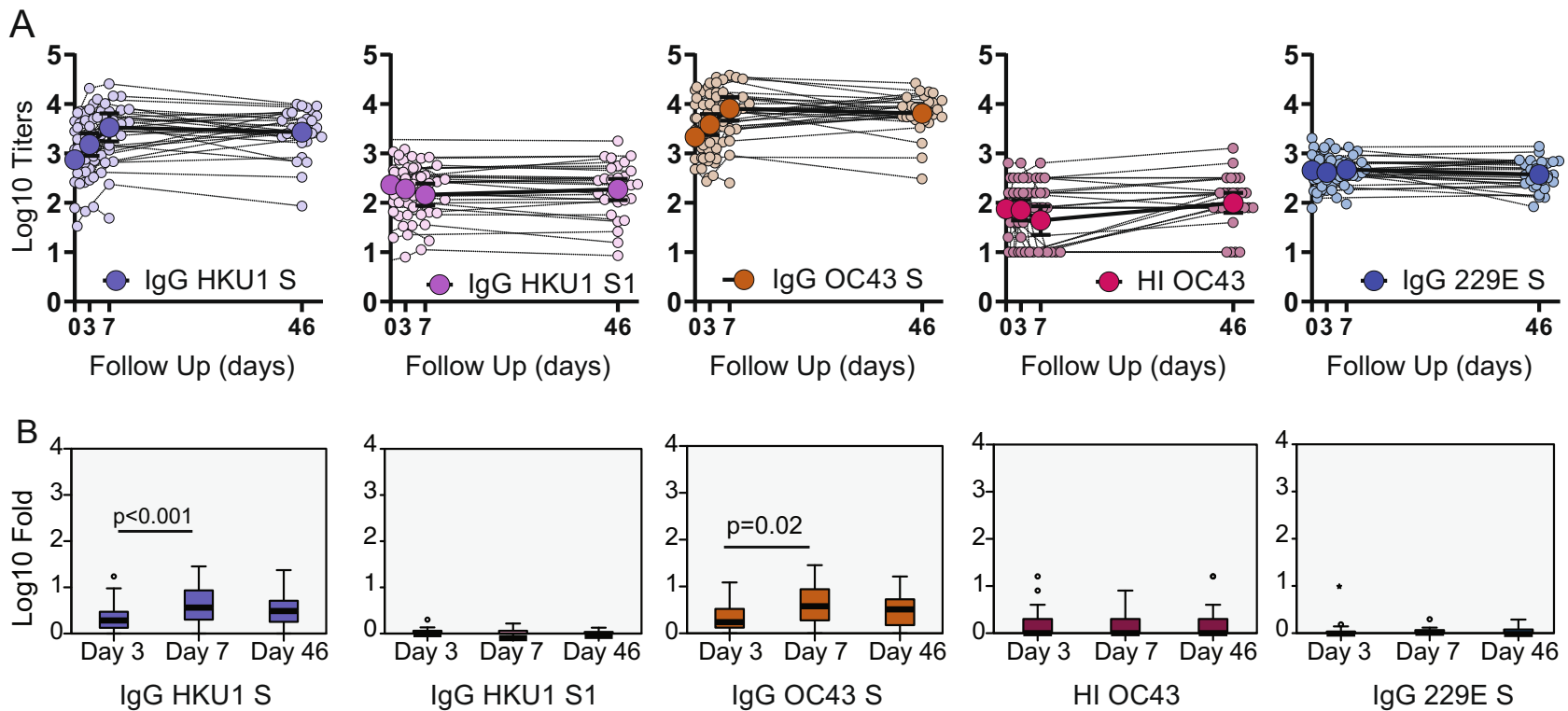

Fig. 3 Longitudinal antibody response to selected seasonal human coronaviruses antigens. Serum from hospitalized COVID-19 patients was analyzed at baseline, at hospital recruitment and days 3 and 7. A subsequent sample was collected in the convalescence period in the COVID-19 survivors with mean time of 46 days. A Longitudinal profile of antibodies against betacoronavirus (HKU1 and OC43) and alphacoronavirus (229E) antigens. Antibody titer was quantified as area under the curve (AUC) after serial serum dilution for each sample (Supplementary Fig. 1). Calculated AUC at each time is shown to quantify changes over time for each individual (small dots) against immunoglobulin G (IgG) HKU1 spike, IgG HKU1 S1, IgG OC43 spike and IgG 229E. Geometric mean titer (GMT, big dots) and confidence interval (CI 95\%) are also shown. Hemagglutination inhibition (HI) assay were also performed for OC43 and GMT of end point titers are shown at each time point. B Boxplot diagram of geometric mean fold rise (GMFR) antibody titers against seasonal coronaviruses at the same time points: IgG HKU1 spike, IgG HKU1 S1, IgG OC43 spike and IgG 229E; and HI titer. Related-samples Friedman's two-way ANOVA was performed. Significant adjusted $p$ values after pairwise comparisons are shown for each comparison. Black bar indicates GMFR values, box indicates IQR (Q1-Q3), lines indicate minimum and maximum. Outliers from the observed distribution are shown. Total $n=116$ biologically independent serum samples (day $0=37$, day $3=29$, day $7=22$, day $46=28$ ). $n=116$ biological samples examined against four different seasonal coronavirus substrates for ELISA assays; ELISAs for each substrate were run once each. $N=116$ serum samples examined over two independent experiments for hemagglutination assays.

HKU1 pre-existing immunity on induction of neutralizing antibodies against SARS-CoV-2 in COVID-19 patients over time (Fig. 6A). A similar trend was found for the levels of pre-existing antibodies against the OC43 spike (Fig. 6B). Interestingly, the impact of back-boosting on IgM against the $\mathrm{S}$ protein was smaller when compared to IgG S or RBD. Scatterplots and the predicted regression lines for the relationship of induction of antibodies against SARS-CoV-2 and pre-exposure to betacoronaviruses are shown in Fig. 5A-D according to time points in the longitudinal follow-up. To assess neutralization potency according to the levels of pre-existing levels of seasonal coronaviruses, we normalized levels of IgG against seasonal human coronavirus antigens by the levels of anti- spike IgG from SARS-CoV-2 virus at the same time points. This analysis allows for comparison of high vs. low presence of pre-existing antibodies toward OC43, 229E, or HKU1 (antibodies at baseline) against the elicitation of SARS-CoV-2 antibodies over the observation course. We then tested whether those patients with higher hCoV/SARS-CoV-2 IgG ratio had lower induction of neutralizing antibodies. After linear regression analysis some disparities were found (Fig. 7). In general, the higher hCoV/SARS-CoV-2 IgG ratio for HKU1 and OC43 IgG S at baseline and day 3, the lower was the induction of antibodies with neutralizing activity to SARS-CoV-2, suggesting some limitations for the ability to elicit robust protective antibody responses against novel antigenic epitopes of SARS-CoV-2 in patients with high levels of cross-reactive antibodies against circulating betacoronaviruses.

Finally, and to test whether imprinting on B cell compartment could also influence antibody responses against more divergent mutated spike proteins from SARS-CoV-2 variants, we measured antibody responses against the spike protein of two SARS-CoV-2 variants. These variants, B.1.1.7 and B.1.351 emerged in late 2020 in United Kingdom and South Africa, respectively. Both B.1.1.7 and B.1.351 bear a N501Y mutation within the RBD while B.1.351 contains also K417N, E484K changes. In addition, further mutations can be found outside of the RBD domain. We performed ELISA against the B.1.1.7 and B.1.351 RBDs as well as neutralization assays against the authentic hCoV-19/England/ 204820464/2020 (B.1.1.7) and hCoV-19/South Africa/KRISPK005325/2020 (B.1.351) variants. Interesting, when percentage of decrease compared to the reference was calculated, we found that responses targeting the RBD dropped from 50 to almost $100 \%$ for B.1.1.7 and B.1.351, respectively (Fig. 8A). In contrast, neutralizing titers against B.1.1.7 were similar to USA-WA1/2020, while percentage of decrease respect to B.1.351 was around $50 \%$, indicating presence of neutralizing antibodies directed against epitopes different to those contained in the RBD, such as those directed against the N-terminal domain (Fig. 8B). Finally, we calculated Pearson correlation coefficients to examine the relationship between seasonal coronavirus HKU1 and OC43 pre-existing immunity and the ELISA antibody responses against the mutated RBDs. Pearson correlation matrices in Fig. 8C, D shows the relationship between pre-existing antibody levels against OC43 and HKU1 and fold induction against RBDs containing N501Y only, or N501Y, K417N, and E484K mutations. No significant correlation was found between pre-exposure to seasonal coronaviruses and responses against the mutated RBDs. The BACO cohort presented in here was enrolled in the first wave of SARS-CoV-2 in Spain, and the likelihood of being infected against a similar variant to Wuhan-Hu-1 is high. It is 
A
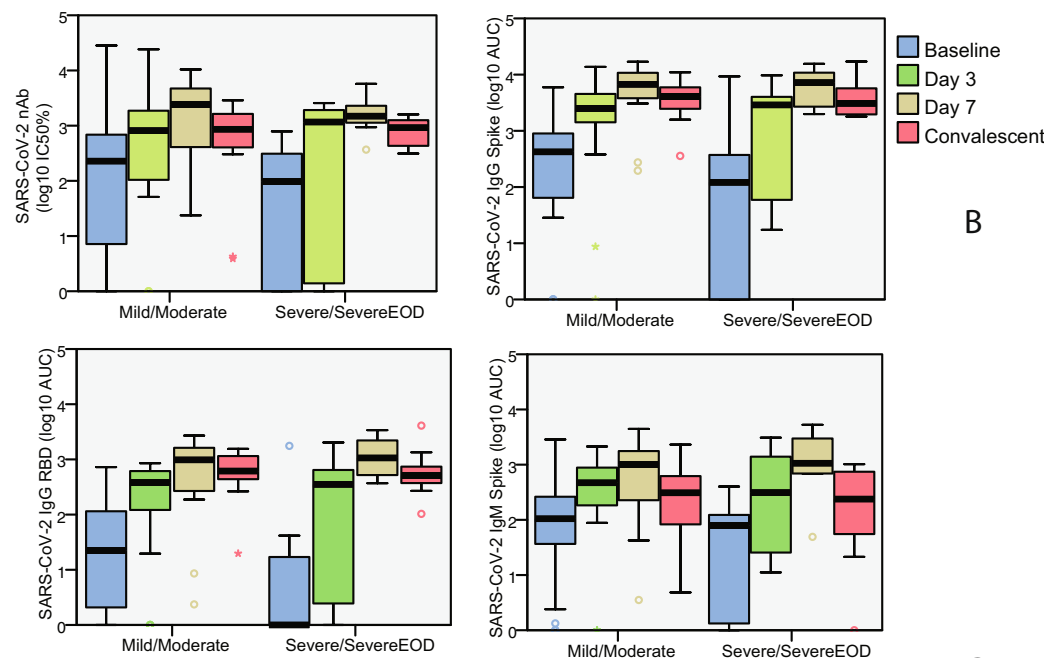

B
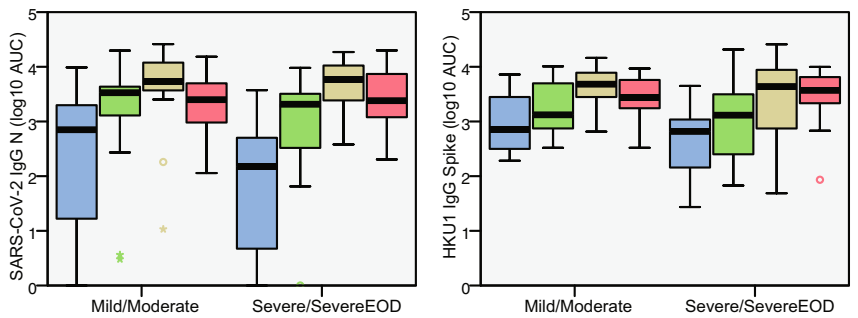

C

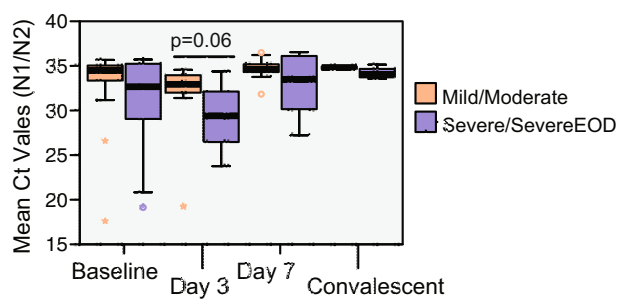

\section{SARS-CoV-2}

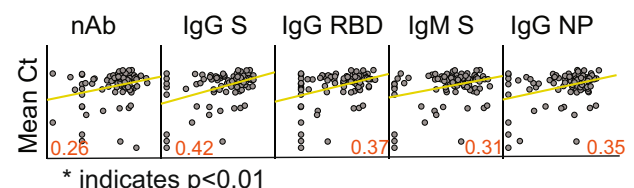

indicates $p<0.01$
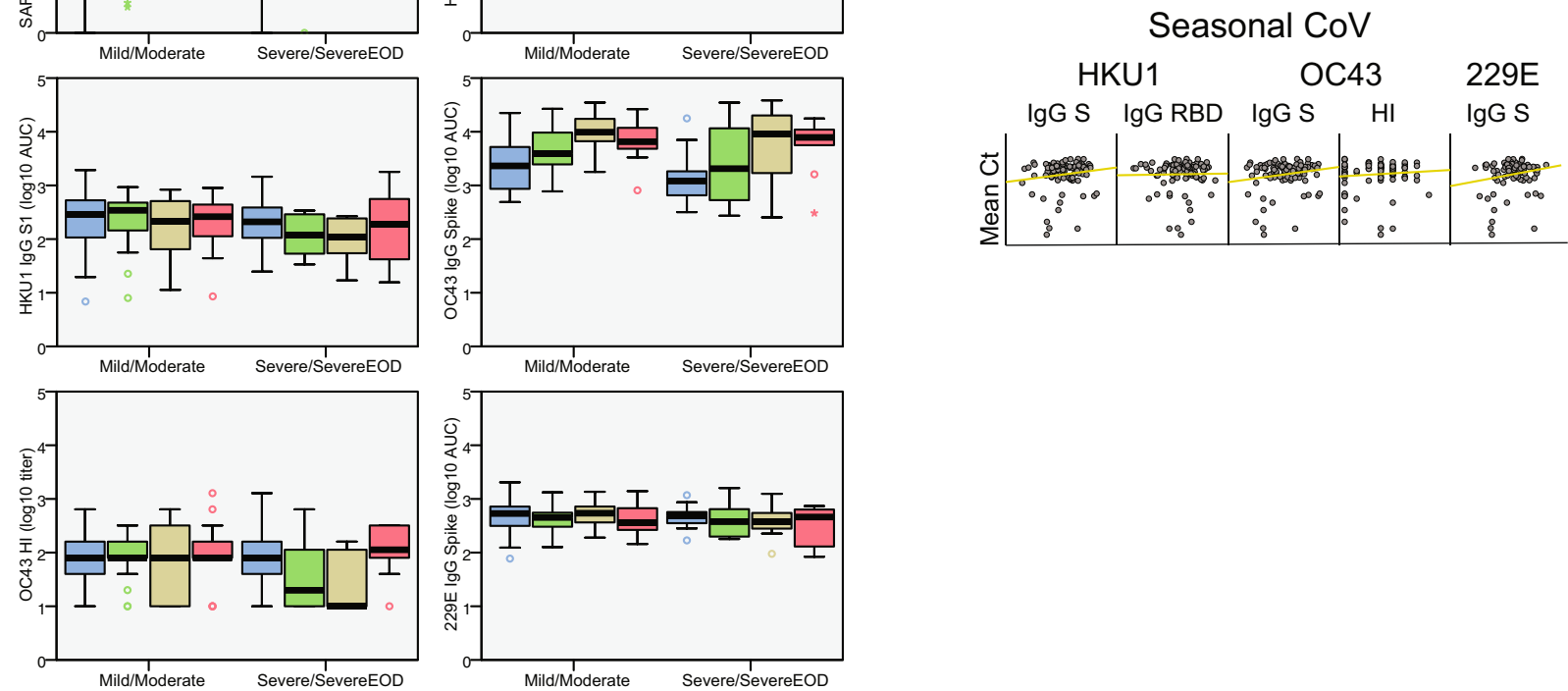

Fig. 4 Antibody response according to disease severity and viral loads in the BACO cohort. A Boxplot diagram of ELISA as area under the curve (AUC) titers against SARS-CoV-2 and endemic human coronaviruses at each time point in mild/moderate vs. severe COVID-19: IgG spike, IgG RBD, IgM spike and IgG NP; and neutralizing titer (IC50\%); and HKU1 IgG spike, HKU1 IgG S1 subunit, OC43 IgG spike and 229E IgG spike; and OC43 hemagglutination titers. Black bar indicated median values, box indicates IQR (Q1-Q3), and lines indicate minimum and maximum. Outliers from the observed distribution are shown when present in each case. Total $n=116$ biologically independent serum samples (day $0=37$, day $3=29$, day $7=22$, day $46=28$ ). $n=116$ biological samples examined against eight different SARS-CoV-2 and seasonal coronavirus substrates for ELISA assays; ELISAs for each substrate were run once each. $N=116$ serum samples examined over two independent experiments for neutralization and hemagglutination assays. B Boxplot diagram of mean threshold cycle (Ct) values in mild/moderate vs. severe COVID-19 during the follow-up. N protein was detected by RT-qPCR. Black bars indicate median values, the box indicates IQR (Q1-Q3), and lines indicate minimum and maximum. Outliers from the observed distribution are shown when present in each case. Total $n=93$ biologically independent nasopharyngeal swab (day $0=37$, day $3=28$, day $7=22$, day $46=6$ ). $n=93$ biological samples examined against two different SARS-CoV-2 primers over two independent experiments each. Mann-Whitney $U$ test for independent samples was performed. Reported $p$ values are based on two-tailed tests. C Scatterplot of the relationship between measured SARS-CoV-2 and seasonal coronaviruses antibody responses and Ct values in the COVID-19 patients. Pearson coefficient of statistically significant correlations is indicated in red. Matrix axis are $\log 10$ values scaled from 0 to 4 . Total $n=93$ biologically independent nasopharyngeal (NP) swab and 93 paired serum samples. Pearson correlation was calculated based on matched NP and serum samples. $P$ values for statistically significant values are shown and based on two-tailed tests. Source data are provided as a Source Data File. 
A

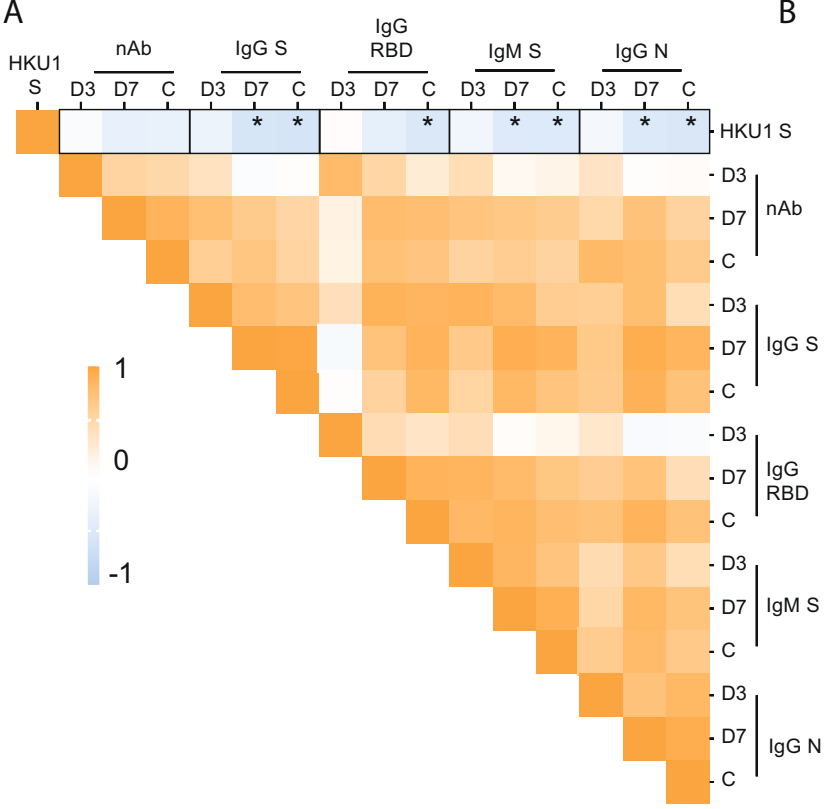

B

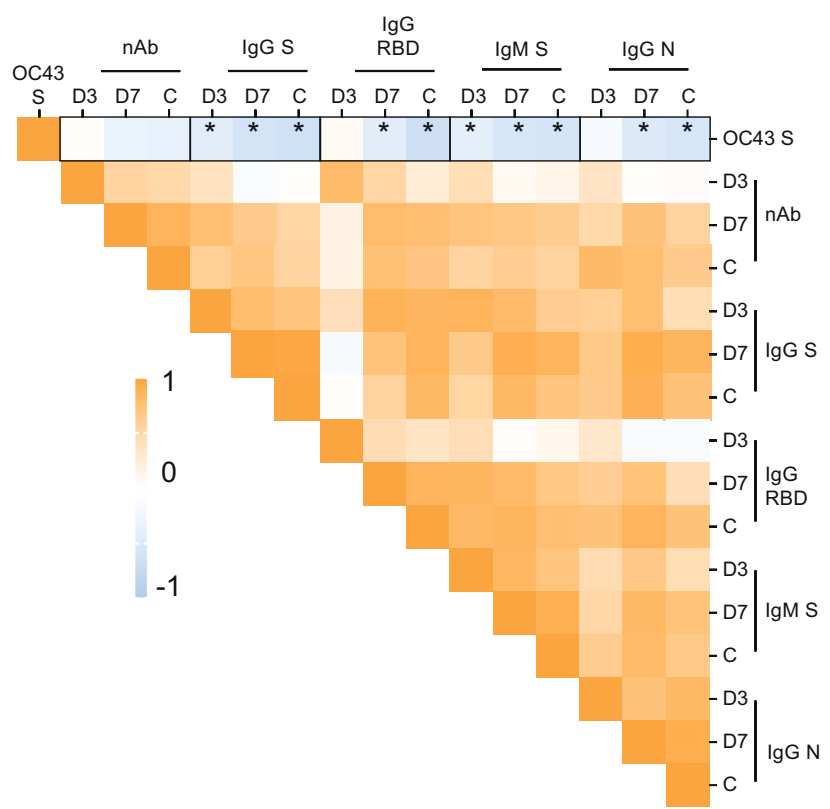

C

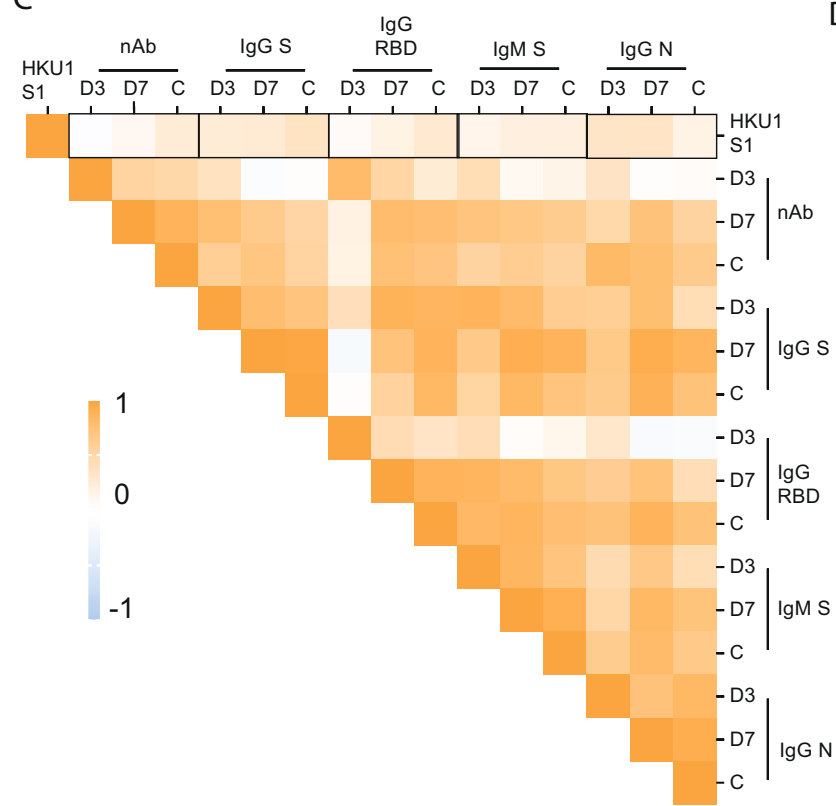

D

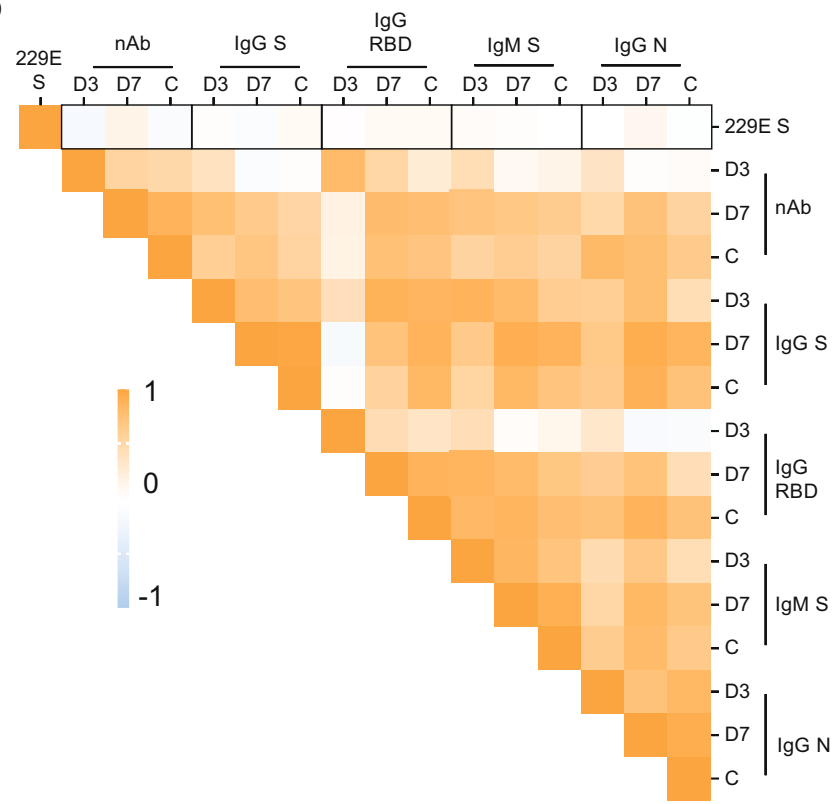

Fig. 5 Immunological imprinting on SARS-CoV-2 antibody response. A, B Heat map of Pearson correlation matrices between pre-existing levels of seasonal hCoV (A IgG HKU1 S; and B IgG OC43 S) and fold induction of SARS-CoV-2 antibodies at each time point: neutralizing (nAb), IgG spike, IgG RBD, IgM spike and IgG NP. Statistically significant correlations in the underlined intersections are indicated with asterisk ( ${ }^{*}$ ); D3: day 3; D7: day 7; C: convalescence. C, D Scatterplot of baseline IgG levels for HKU1 and OC43 S protein and fold induction of SARS-CoV-2 antibodies: neutralizing (nAb), IgG spike, IgG RBD. Overlay shows relationship with induction of de novo antibodies against SARS-CoV-2 at each time point. Fitted linear regression and standardized beta coefficient ( $95 \%$ confidence interval, $\mathrm{Cl}$ ) for significant linear regressions are shown.

likely that the drop on RBD titers for the variants is responsible for the lack of detection of an imprinting effect with these variants.

\section{Discussion}

Our findings provide a dynamic characterization of the antibody response to SARS-CoV-2 in COVID-19 patients and provide evidence of immune imprinting in these patients. Our results demonstrate back-boosting in the BACO cohort against the conserved epitopes of the spike protein of OC43 and HKU1 betacoronaviruses. No induction was detected for the variable regions of these viruses, such as the S1 domain, or to more divergent seasonal alphacoronaviruses, such as 229E. Although antibody cross-reactivity has been reported in cross-sectional studies $^{22,23,25,32}$, our cohort has allowed for quantification and detailed representation of the longitudinal outcome of the immune response by taking into consideration past exposure to related antigens. Neutralization activity of antibodies might be used as a proxy for protection against SARS-CoV- 2 infection ${ }^{46,47}$. IgG responses to the spike and RBD of SARS-CoV-2 showed persistence over the time period of our study with slight changes in antibody levels in convalescent sera as compared to the peak of 
A

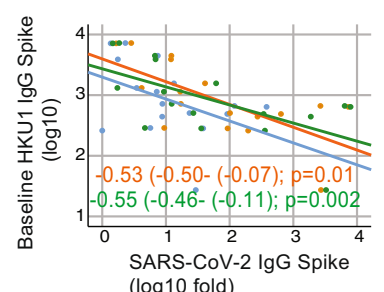

( $\log 10$ fold)

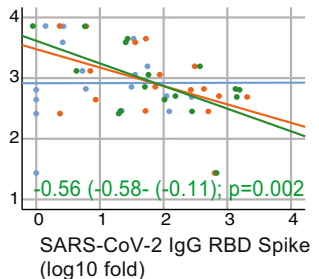

$(\log 10$ fold)

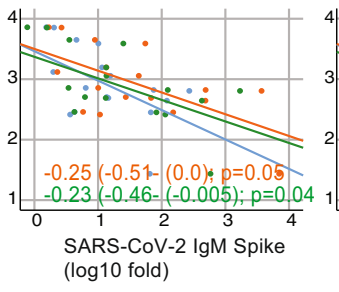

( $\log 10$ fold)

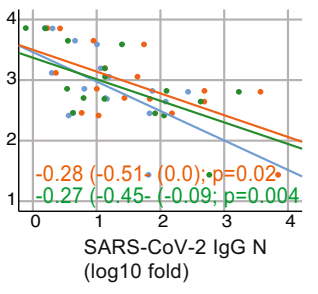

$(\log 10$ fold)

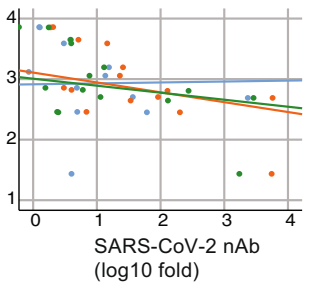

(log10 fold)

B

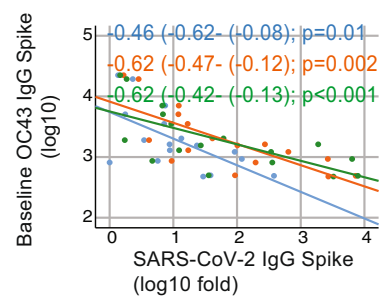

Day 3

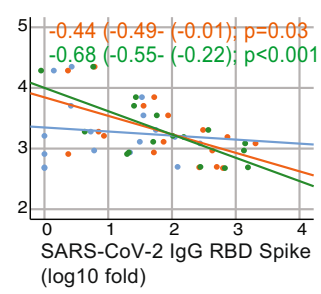

Day 7

Convalescence
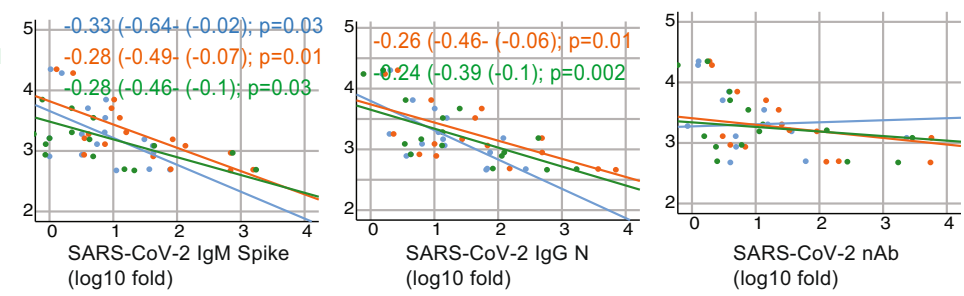

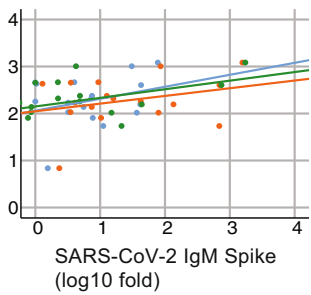

$(\log 10$ fold $)$

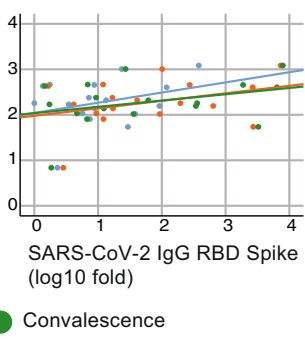

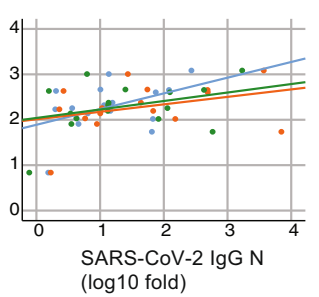

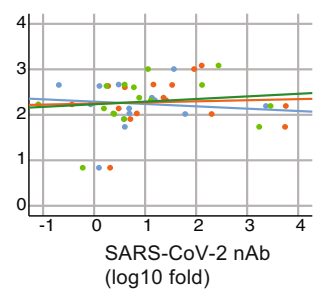

Day 3

Day 7

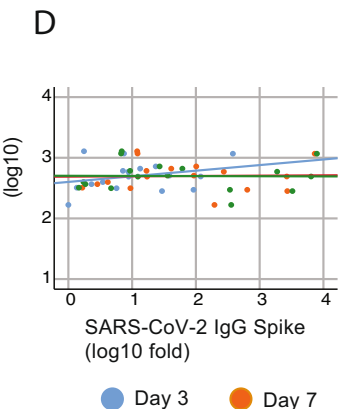

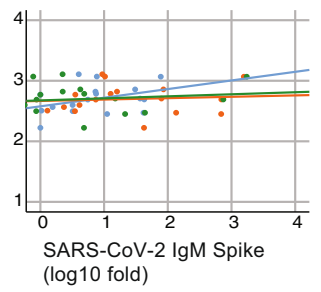

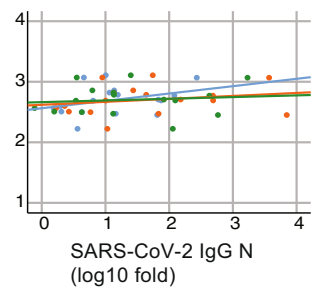

Fig. 6 Cross-reactivity with conserved epitopes against selected betacoronaviruses predicts negative influence on de novo anti-SARS-CoV-2 antibody responses. A-D Scatterplot of baseline IgG levels for HKU1, OC43 and 229E S protein; and HKU1 S1 and fold induction of SARS-CoV-2 antibodies: neutralizing (nAb), IgG spike, IgG RBD, IgM spike, and nucleoprotein. Overlay shows relationship with induction of de novo antibodies against SARS-CoV-2 at each time point. Fitted linear regression and standardized beta coefficient ( $95 \%$ Confidence Interval, $\mathrm{Cl}$ ) for significant linear regressions are shown. Reported $p$ values are based on two-tailed tests.

antibody induction at day 7. Importantly, immunity to other betacoronavirus spikes, like HKU1 and OC43, limited the induction of de novo responses to all SARS-CoV-2 antigens tested. All patients also developed detectable levels of spike IgG/ IgM and N IgG. Although no significant correlation was found between pre-exposure to seasonal coronaviruses and induction of protective antibodies with neutralizing activity, simple linear regression estimated a negative relationship, and the predicted line approximated a negative influence on development of de novo neutralizing antibodies over time. Similarly, baseline antibody levels to HKU1 or OC43 spike after SARS-CoV-2 IgG levels normalization limited the induction of neutralizing antibody levels after in the follow-up.

While we could not find statistically significant differences for antibody levels in patients with mild vs. severe disease, the latter showed a delay in antibody responses. Moreover, anti-SARSCoV-2 antibodies inversely correlated with viral loads in respiratory samples, whereas virus clearance could not be linked to back-boosting of antibodies toward the S2 subunit of the seasonal human coronaviruses. Importantly, several reports have shown cross-reactivity between pre-existing memory $\mathrm{T}$ cells to seasonal coronaviruses and SARS-CoV-2 $2^{25,48}$ pointing to a 
A

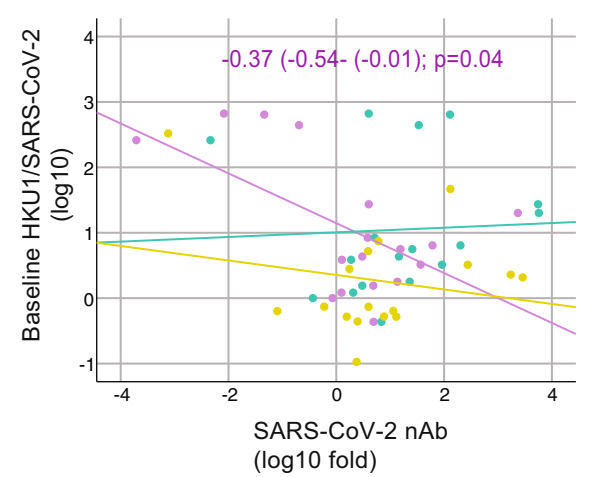

C

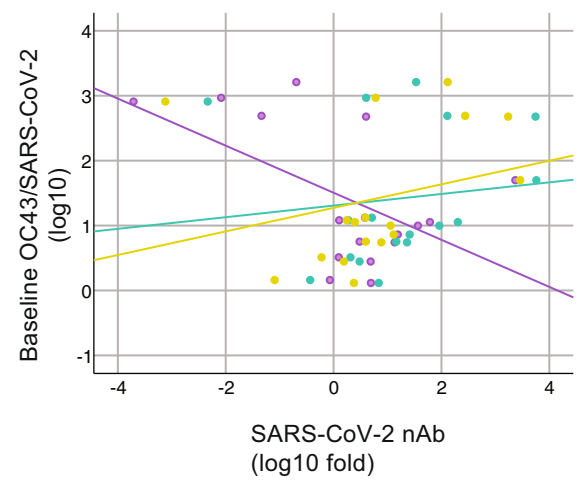

B

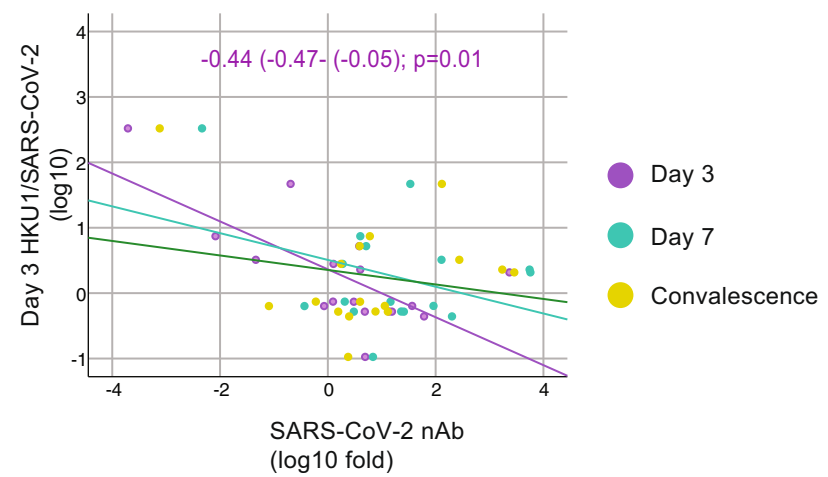

D

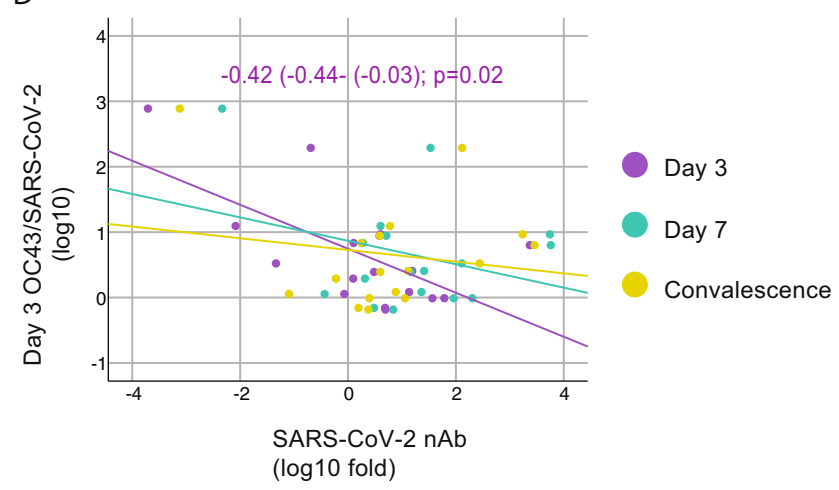

Fig. 7 Influence of levels of back-boosting to HKU1 and OC43 normalized by levels of anti- SARS-CoV-2 antibodies on neutralizing antibodies induction. A-D Scatterplot of baseline and day 3 lgG levels for HKU1 and OC43 S protein normalized by the levels of SARS-CoV-2 IgG and their relationships with fold induction of SARS-CoV-2 neutralizing antibodies ( $n A b)$ over time. Overlay shows linear regression at each time point. Fitted linear regression and standardized beta coefficient ( $95 \%$ confidence interval, $\mathrm{Cl}$ ) for significant regression are shown. Reported $p$ values are based on twotailed tests.

potential role of heterologous immunity as an additional mechanism of protection or even differences on COVID-19 outcomes. However, our results allow for a contrasting hypothesis in which early priming of the memory B cell compartment due to pre-exposure to seasonal coronaviruses could dampen secondary responses toward new epitopes of SARS-CoV-2. Nonetheless, all patients from the BACO cohort developed antibody responses against SARS-CoV-2 antigens and specific neutralizing antibodies. In addition, SARS-CoV-2 is evolving, and some variants including 501Y spike mutations have emerged and rapidly spread in countries, such as UK, South Africa, and Brazil (https://www. who.int/csr/don/31-december-2020-sars-cov2-variants/en/).

These variants contain mutations that introduce amino acid changes in RBD residues targeted by neutralizing antibodies and therefore have functional significance. There is a general concern on whether new emerging variants (also known as variants of concern, VOC) could evade immunity generated not only by previous infections but also vaccination causing a drop on the effectiveness of COVID-19 vaccines. It is possible that firstgeneration COVID-19 vaccines will need to be updated according to the circulating variants in the future.

Our observation has important impact of on the development of COVID-19 vaccines and the potential interactions with preexisting immunity should be taken into consideration in the path to optimal vaccines. COVID-19 vaccines in use aim at the induction of responses against the full-length $S$ protein of SARS-CoV-2 49 , which is known to contain cross-reactive nonneutralizing epitopes that are shared with seasonal human betacoronaviruses. A similar scenario to our studies in infected people could be proposed for the vaccines, with some differences due to the nature of the stimulus itself. Back-boost of cross-reactive antibody responses might lead to less protective antibodies directed against non-neutralizing conserved epitopes between the $S$ antigen of the vaccine and the $S$ proteins of seasonal human betacoronaviruses ${ }^{50}$. On the other hand, it is also possible that cross-reactive antibodies provide protection from severe disease outcomes by immune mechanisms of action different from those involved on in vitro virus neutralization, such as antibodydependent cytotoxicity. That is the case for broadly cross-reactive and non-neutralizing anti-influenza antibodies targeting the conserved stalk domain of the hemagglutinin protein of influenza viruses. HA stalk antibodies can mediate antibody-dependent cell cytotoxicity, contributing to protection from disease severity independently of neutralizing activity ${ }^{51}$. Whether in vitro nonneutralizing anti-SARS-CoV-2 antibodies contribute to protection or disease or are neutral is still not clear.

Our study has several limitations. We comprehensively characterized antigen specificity, neutralization potency, and viral cross-reactivity against multiple coronaviruses over time. However, the number of subject enrolled remained relatively small due to the challenges and restrictions faced by the hospitals during the initial spread of SARS-CoV-2, underpowering the conclusions of this study. In addition, all the patients enrolled required hospitalization, and the pre-existing immunity of asymptomatic or mild cases of COVID-19 could not be characterized in this study. Still, our results demonstrate that the antibody response against SARS-CoV-2 infection and, potentially vaccination, is influenced by imprinting of the $\mathrm{B}$ cell compartment due to previous 


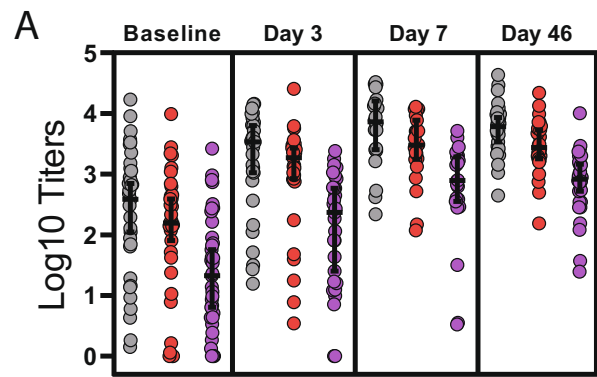

IgG Titers

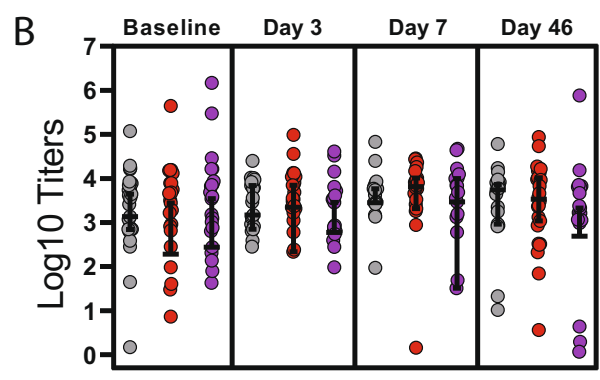

nAb Titers

C

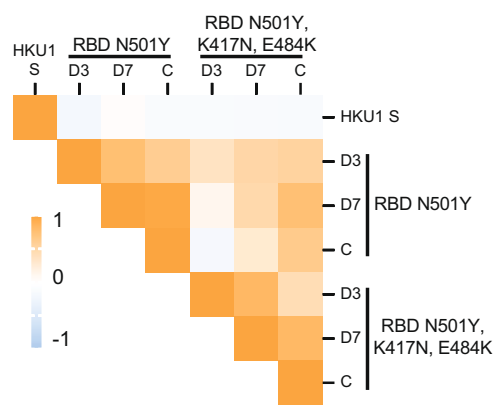

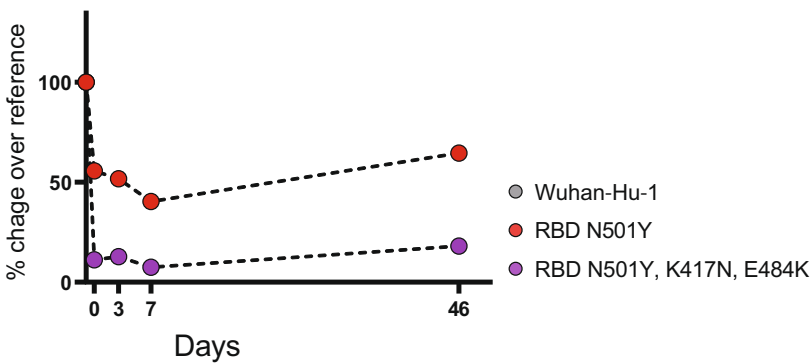
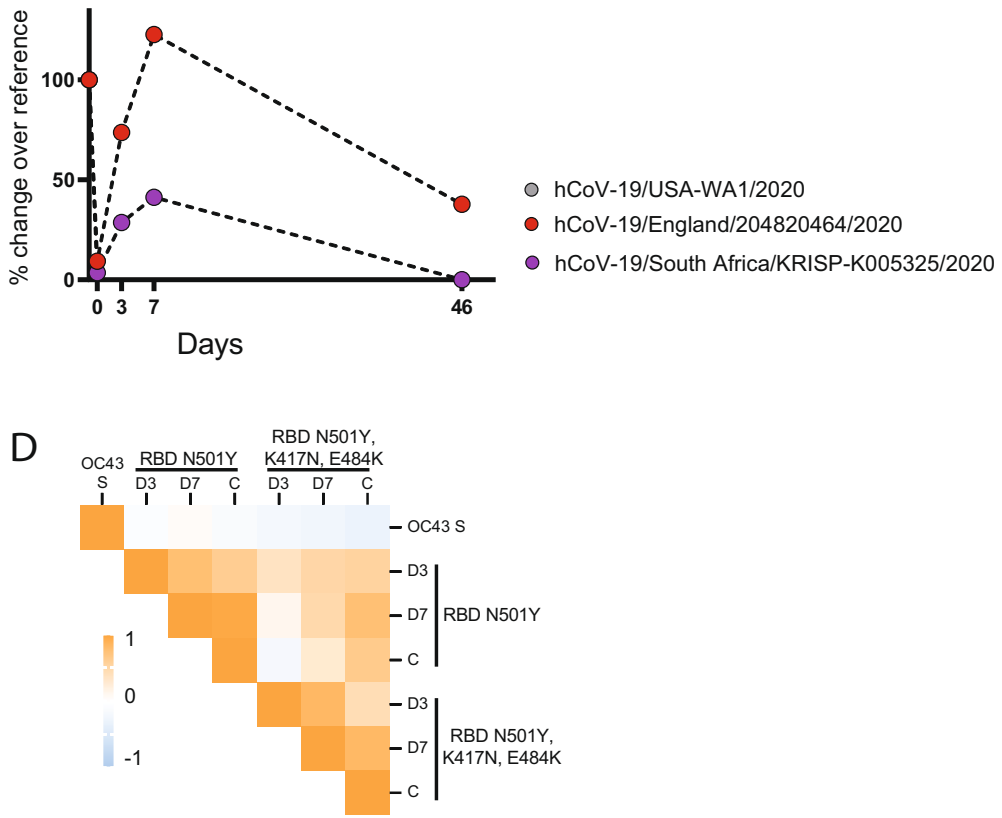

Fig. 8 Imprinting and antibody response against emerging variants of SARS-CoV-2. A ELISA against the receptor-binding domains (RBDs) of WuhanHu-1 (reference), and mutated RBDs representative of UK (N501Y) and South African (K417N, E484K and N501Y) variants. B Neutralizing titers against the authentic hCoV-19/England/204820464/2020 (B.1.1.7) and hCoV-19/South Africa/KRISP-K005325/2020 (B.1.351). Errors indicate geometric mean titer (GMT) and confidence interval $(\mathrm{Cl} 95 \%)$ at each time point for ELISA against each RBD or neutralizing titers against each variant. Percentage of decrease titers compared to reference has been calculated and data are shown in the right for ELISA and nAb titers. Total $n=116$ biologically independent serum samples (day $0=37$, day $3=29$, day $7=22$, day $46=28$ ). $n=116$ biological samples examined against three different SARS-CoV-2 RBDs; ELISAs for each substrate were run once each. $N=116$ serum samples examined for three different SARS-CoV-2 variants over two independent experiments each. C-D Heat map of Pearson correlation matrices between pre-existing levels of seasonal CoVs: IgG HKU1 S; and B IgG OC43 S; and fold induction of antibodies against RBD N501Y and RBD N501Y, K417N, E484K RBD at each time point. D3 day 3, D7 day 7, C convalescence.

exposure to seasonal human betacoronaviruses. This is consistent with additional recent studies ${ }^{33}$. It will be important to investigate the potential functional consequences of this imprinting in the induction of protective immune responses after SARS-CoV-2 infection and vaccination in the long term, and in the very likely case that the current pandemic evolves into epidemic outbreaks.

\section{Methods}

Experimental model and subject details: The BACO cohort. An observational prospective human cohort study of COVID-19 was carried out during the first pandemic wave (March-May 2020) of SARS-CoV-2 in Barcelona (Spain) and was termed the BACO Cohort. A positive case was defined according to international guidelines when a nasopharyngeal (NP) swab tested positive for SARS-CoV-2 by reverse transcriptase quantitative polymerase chain reaction (RT-qPCR) upon hospital admission. All patients or their legally authorized representatives provided informed consent. Serum and samples were collected at the enrollment in the study (baseline), and at days 3 and 7 post enrollment. A convalescence sample was collected from survivors after recovery and hospital discharge with a mean time of 46 days (range, 30-56 days). The total number of serum samples was 116 . Data on demographics, including age and sex, comorbidities, clinical signs and symptoms, interventions, and outcomes are described in Table 1. Severity of COVID-19 was assigned following a described severity scale based on oxygen saturation $\left(\mathrm{SpO}_{2}\right)$, presence of pneumonia/imaging, oxygen support defined as use of high-flow nasal cannula (HFNC), non-rebreather mask (NRB), bilevel positive airway pressure (BIPAP) or mechanical ventilation (MV); and kidney (creatinine clearance, $\mathrm{CrCl}$ ) and liver (alanine aminotransferase, ALT) function ${ }^{44}$ : mild (SpO2 $>94 \%$ AND no pneumonia), moderate ( $\mathrm{SpO} 2<94 \% \mathrm{AND} / \mathrm{OR}$ pneumonia), severe (use of HFNC, NRB, BIPAP or MV AND no vasopressor use AND $\mathrm{CrCl}>30$ AND ALT $<5 \mathrm{x}$ upper limit of normal) and severe with end-of organ disease (Use of HFNC, NRB, BIPAP or MV AND vasopressor use $\mathrm{OR} \mathrm{CrCl}>30$ or new $\mathrm{HD}$ OR ALT $<5 \mathrm{x}$ upper limit of normal).

The study protocol was approved by the Institutional Review Board of University Hospital of Bellvitge, Barcelona, Spain; and by the Icahn School of Medicine at Mount Sinai, New York, US.

Cell lines. Vero E6 cells were originally purchased from the American Type Culture Collection (ATCC, Cat\# CRL-1586). Cells were maintained in Dulbecco's modified Eagle's medium (DMEM) w/ L-glutamate, sodium pyruvate (Corning) supplemented with $10 \%$ fetal bovine serum (FBS), $10 \mathrm{U}$ penicillin per $\mathrm{ml}$, and $10 \mathrm{mg}$ streptomycin per ml. HCT-8 human cells line was obtained from the ATCC (Cat\#CCL-24) and maintained in Roswell Park Memorial Institute 1640 medium (Gibco) supplemented with $10 \% \mathrm{FBS}, 10 \mathrm{U}$ penicillin per $\mathrm{ml}$, and $10 \mathrm{mg}$ 
streptomycin per ml. Cell lines were supplemented with Normocyn (Invivogen, Cat. ant-nr-1) to prevent Mycoplasma contamination.

Virus strains. SARS-CoV-2, isolate USA-WA1/2020, was initially obtained from BEI Resources (Cat\#NR-52281) and further propagated in Vero E6 cells ${ }^{52}$. Human coronavirus OC43 was obtained from the ATCC (Cat\#VR-1558) and propagated on HCT-8 cells following ATCC recommendations.

\section{Microneutralization assays. Microneutralization (MN) assays for antibody} characterization were performed as described ${ }^{52}$. Briefly, Vero E6 cells were seeded in a 96-well cell culture plate with complete Dulbecco's Modified Eagle Medium (cDMEM)(Corning) [Penicillin-streptomycin (Corning), non-essential amino acids (Corning), 10\% FBS (Peak)]. The following day, heat-inactivated serum samples were serially diluted three-fold in $1 \mathrm{x}$ minimum essential medium with $2 \%$ FBS with a final volume of $200 \mu \mathrm{l} .80 \mu \mathrm{l}$ of serum dilution was transferred to a new 96-well plate and 600 Tissue Culture Infectious Dose 50 percent per well of SARS-CoV-2 $(80 \mu \mathrm{l} /$ well $)$ and mixed with serum dilution and incubated for $1 \mathrm{~h}$ at $37^{\circ} \mathrm{C}$. Then, cDMEM was removed from Vero e 6 cells and $120 \mu \mathrm{l}$ of virus-serum mixture was added to the cells. The cells were incubated at $37^{\circ} \mathrm{C}$ for $1 \mathrm{~h}$. Virus-serum mixture was removed from the cells and $100 \mu \mathrm{l}$ of serum dilutions and $100 \mu \mathrm{l}$ of $1 \mathrm{xMEM}$ with $2 \%$ FBS was added to the cells. The cells were incubated for $24 \mathrm{~h}$ and then fixed with $10 \%$ paraformaldehyde (Polysciences) for $24 \mathrm{~h}$ at $4{ }^{\circ} \mathrm{C}$. Following fixation, the cells were washed with phosphate-buffered saline (Corning) with tween20 (Fisher) (PBST) and permeabilized with $0.1 \%$ Triton X-100 (Fisher) for $15 \mathrm{~min}$ at room temperature. The cells were washed three times using PBST and blocked with $3 \%$ milk in PBST for $1 \mathrm{~h}$ at room temperature. Then, the cells were incubated with mouse antibody 1C7 (anti-SARS N antibody, kindly provided by Dr. Moran) at a dilution of 1:1000 in 1\% milk in PBST and incubated for $1 \mathrm{~h}$ at room temperature. The cells were washed three times with PBST. Then, the cells were incubated with goat anti-mouse IgG-HRP (Abcam, Cat. ab6823) at a dilution of $1: 10,000$ in $1 \%$ milk in PBST and incubated for $1 \mathrm{~h}$ at room temperature. The cells were washed three times with PBST and TMBE Elisa peroxidase substrate (Rockland) was added. After $15 \mathrm{~min}$ incubation, sulfuric acid $4.0 \mathrm{~N}$ (Fisher) was added to stop the reaction and the readout was done using a Synergy H1 plate reader (BioTek) at an OD450.

Recombinant proteins. The recombinant spike protein and recombinant RBD of SARS-CoV-2 were generated and expressed as previously described in detail ${ }^{52,53}$. In brief, the mammalian cell codon-optimized nucleotide sequence for the soluble version of the spike protein (amino acids 1-1213) including a C-terminal thrombin cleavage site, signal peptide, hexahistidine tag and $\mathrm{T} 4$ foldon trimerization domain were cloned into pCAGGS mammalian expression vector. The sequence of the spike protein was additionally modified to remove the polybasic cleavage site and two proline residues introduced to increase protein stability. The nucleotide sequence for the RBD (amino acids 319-541) including a signal peptide was cloned into pCAGGS. RBD mutants were generated in the pCAGGS RBD construct by changing single residues using site-directed mutagenesis. The expression plasmids encoding for the spike of common human coronavirus 229E, OC43, and HKU1 were obtained from the NIH (kindly provided by Kizzmekia Corbett and Barney Graham) and the expression plasmid encoding for SARS-CoV-2 NP was constructed at Mount Sinai. The recombinant proteins were expressed in Expi293F cells (Thermo Fisher) using the ExpiFectamine 293 Transfection Kit (Thermo Fisher) according to the manufacturer's protocol. Cell supernatant was harvested, and the proteins purified using Ni-NTA Agarose (Qiagen). The proteins were concentrated in Amicon centrifugal units (EMD Milipore) and correct size confirmed by reducing sodium dodecyl sulfatepolyacrylamide gel electrophoreses. The recombinant S1 subunit of HKU1 was purchased from Sino Biological (Cat. 40021-V08H).

Enzyme-linked immunosorbent assay (ELISA). Ninety-six-well microtiter plates (Thermo Fisher) were coated with $50 \mu \mathrm{L}$ recombinant protein (RBD, SARS-CoV-2 full-length spike, SARS-CoV-2 NP, OC43 spike, 229E spike, or HKU1 spike, respectively) at a concentration of $2 \mu \mathrm{g} / \mathrm{mL}$ overnight, $4{ }^{\circ} \mathrm{C}$. The next day, the plates were washed three times with PBS (phosphate-buffered saline; Gibco) containing $0.1 \%$ Tween-20 (T-PBS, Fisher Scientific) using an automatic plate washer (BioTek). After washing, the plates were blocked for $1 \mathrm{~h}$ at room temperature with 200 $\mu \mathrm{l}$ blocking solution (PBS-T with $3 \%(\mathrm{w} / \mathrm{v})$ milk powder (American Bio)) per well. The blocking solution was removed and serum samples diluted to a starting concentration of $1: 80$, serially diluted $1: 3$ in PBS-T supplemented with $1 \%(\mathrm{w} / \mathrm{v})$ milk powder and incubated at room temperature for $2 \mathrm{~h}$. The plates were washed three times with PBS-T and $50 \mu \mathrm{l}$ anti-human IgG (Fab-specific) horseradish peroxidase antibody (HRP, Sigma, Cat. A0293) diluted 1:3,000 in PBS-T containing $1 \%$ milk powder was added to all wells and incubated for $1 \mathrm{~h}$ at room temperature. The plates were washed three times using the plate washer and $100 \mu \mathrm{L}$ SigmaFast ophenylenediamine dihydrochloride (Sigma) was added to all wells for $10 \mathrm{~min}$. The enzymatic reaction was stopped with $50 \mu \mathrm{L} 3 \mathrm{M}$ hydrochloric acid (Thermo Fisher) per well and the plates read at a wavelength of $490 \mathrm{~nm}$ with a plate reader (BioTek). The results were recorded in Microsoft Excel and AUC values were computed by plotting normalized OD values against the reciprocal serum sample dilutions for ELISAs in GraphPad Prism.
Hemagglutination inhibition (HAI) assay. Serum samples were incubated overnight with receptor-destroying enzyme (RDE; Denka Seiken) for $16-18 \mathrm{~h}$ in a $37^{\circ} \mathrm{C}$ water bath. Three volumes (relative to serum) of $2.5 \%$ sodium citrate solution were added and the resulting solution was heat inactivated at $56^{\circ} \mathrm{C}$ in a water bath $(30$ min). Final serum dilutions were adjusted to 1:10 in PBS. OC43 virus was diluted to a final concentration of $8 \mathrm{HA}$ units $/ 50 \mu \mathrm{L}$ in fluorescent treponemal antibody HA buffer (BD Biosciences). Twofold dilutions of RDE treated serum $(25 \mu \mathrm{L})$ were incubated with equal amount of the virus at $8 \mathrm{HA}$ units $/ 50 \mu \mathrm{L}(30 \mathrm{~min}$, room temperature). Chicken red blood cells (RBCs) (Lampire Biological) at $0.5 \%$ in HA buffer $(50 \mu \mathrm{L})$ were added and incubated $45 \mathrm{~min}$ at $4{ }^{\circ} \mathrm{C}$. The HAI titer was determined by taking the reciprocal dilution of the last well in which serum inhibited the HA of RBCs.

Viral loads and qRT-PCR. To detect SARS-CoV-2 RNA in nasal swabs, a modified version of the CDC 2019-nCoV real-time RT-qPCR was used. Primers and probes were commercially available (Integrated DNA Technologies, cat. 10006713, RUO Kit). SARS-CoV-2 primer and probe sets consisted of two 2019-nCoV-specific sets (N1, N2). A third primer set was used to detect host cellular RNaseP. Reactions were run using the QuantiFast Pathogen RT-PCR + IC Kit (QIAGEN, cat. 211454). A list of all primers used, including the names and sequences, is shown in Supplementary Table 3. Assays were run using USA/WA-1/2020 SARS-CoV-2 RNA as a positive control (20,000 genome copies per reaction) and nuclease-free water as a non-template control in a 384-well format. Reactions were performed in duplicate using the following cycling conditions on the Roche LightCycler 480 Instrument II (Roche Molecular Systems, 05015243001 ): $50^{\circ} \mathrm{C}$ for $20 \mathrm{~min}, 95^{\circ} \mathrm{C}$ for $1 \mathrm{~s}, 95^{\circ} \mathrm{C}$ for $5 \mathrm{~min}$, followed by 45 cycles of $95^{\circ} \mathrm{C}$ for $15 \mathrm{~s}$ and $60^{\circ} \mathrm{C}$ for $45 \mathrm{~s}$. Limit of detection for SARS-CoV-2 was determined by using a commercially available plasmid control (Integrated DNA Technologies, cat. 10006625).

Multiple sequences alignment and conservation scores. MSA to determine the spike protein sequence identity among SARS-CoV-2 (NC_045512.2), and the human endemic betacoronaviruses HKU1 (YP_173238) and OC43

(YP_009555241.1), and alphacoronavirus 229E (NP_073551.1) was performed with ClustalW. Conservation patterns and scores of the spike protein of SARS-CoV-2 were determined using the ConSurf server (https://consurf.tau.ac.il/). Briefly, a MSA of 150 homologous sequences was constructed using MAFFT. Positionspecific conservation scores were computed using an empirical Bayesian algorithm and divided into a discrete scale of nine grades. The conservation scores were projected onto the SARS-CoV-2 spike protein in the closed state (PDB ID 6VXX) as a reference.

Quantification and statistical analysis. All immune assay values were log10transformed to improve linearity. The GMT and 95\% confidence intervals (CI 95\%) were computed by taking the exponent $(\log 10)$ of the mean and of the lower and upper limits of the $95 \%$ CI of the log10-transformed titers. Fold rise was calculated as the ratio between days 3, 7 or convalescent antibody value to baseline levels. GMFR was computed by taking the exponent $(\log 10)$ of the mean fold rise and of the lower and upper limits of the CI $95 \%$ of the $\log 10$-transformed titers. Statistical significance was established at $p<0.05$. All reported $p$ values are based on two-tailed tests. Correlation (Pearson), linear regression, local regression fit-line and relatedsample multiple comparison (Friedman's two-way analysis of variance by ranks, also known as Friedman's two-way ANOVA, and pairwise comparison adjusted by Bonferroni correction) were performed using IBM SPSS Statistics (version 26).

Reporting summary. Further information on research design is available in the Nature Research Reporting Summary linked to this article.

\section{Data availability}

All data are available in the manuscript or the supplementary materials. Source data are provided with this paper. The accession codes for the Structure of the SARS-CoV-2 spike glycoprotein (closed state) EMD: 21452 and PDB: 6VXX. Source data are provided with this paper.

Received: 5 January 2021; Accepted: 25 May 2021; Published online: 18 June 2021

\section{References}

1. Zhu, N. et al. A novel coronavirus from patients with pneumonia in China, 2019. N. Engl. J. Med. 382, 727-733 (2020).

2. Morens, D. M. \& Fauci, A. S. Emerging pandemic diseases: how we got to COVID-19. Cell 182, 1077-1092 (2020)

3. Ieven, M. et al. Aetiology of lower respiratory tract infection in adults in primary care: a prospective study in 11 European countries. Clin. Microbiol Infect. 24, 1158-1163 (2018). 
4. Zaki, A. M., van Boheemen, S., Bestebroer, T. M., Osterhaus, A. D. \& Fouchier, R. A. Isolation of a novel coronavirus from a man with pneumonia in Saudi Arabia. N. Engl. J. Med. 367, 1814-1820 (2012).

5. Peiris, J. S. et al. Coronavirus as a possible cause of severe acute respiratory syndrome. Lancet 361, 1319-1325 (2003).

6. Forni, D., Cagliani, R., Clerici, M. \& Sironi, M. Molecular evolution of human coronavirus genomes. Trends Microbiol. 25, 35-48 (2017).

7. $\mathrm{Bi}$, Q. et al. Epidemiology and transmission of COVID-19 in 391 cases and 1286 of their close contacts in Shenzhen, China: a retrospective cohort study. Lancet Infect. Dis. 20, 911-919 (2020).

8. Jackson, L. A., et al. An mRNA vaccine against SARS-CoV-2 - preliminary report. N. Engl. J. Med. 383, 1920-1931 (2020).

9. Mercado, N. B., et al. Single-shot Ad26 vaccine protects against SARS-CoV-2 in rhesus macaques. Nature 586, 583-588 (2020).

10. van Doremalen, N. et al. ChAdOx1 nCoV-19 vaccine prevents SARS-CoV-2 pneumonia in rhesus macaques. Nature 586, 578-582 (2020).

11. Piccoli, L. et al. Mapping neutralizing and immunodominant sites on the SARS-CoV-2 spike receptor-binding domain by structure-guided highresolution serology. Cell 83, 1024-1042 (2020).

12. Yuan, M. et al. A highly conserved cryptic epitope in the receptor binding domains of SARS-CoV-2 and SARS-CoV. Science 368, 630-633 (2020).

13. Boni, M. F. et al. Evolutionary origins of the SARS-CoV-2 sarbecovirus lineage responsible for the COVID-19 pandemic. Nat. Microbiol. 5, 1408-1417 (2020).

14. Walls, A. C. et al. Structure, function, and antigenicity of the SARS-CoV-2 spike glycoprotein. Cell 183, 1735 (2020).

15. Lachance, C., Arbour, N., Cashman, N. R. \& Talbot, P. J. Involvement of aminopeptidase $\mathrm{N}$ (CD13) in infection of human neural cells by human coronavirus 229E. J. Virol. 72, 6511-6519 (1998).

16. Hulswit, R. J. G. et al. Human coronaviruses OC43 and HKU1 bind to 9-Oacetylated sialic acids via a conserved receptor-binding site in spike protein domain A. Proc. Natl Acad. Sci. USA 116, 2681-2690 (2019).

17. Ge, X. Y. et al. Isolation and characterization of a bat SARS-like coronavirus that uses the ACE2 receptor. Nature 503, 535-538 (2013).

18. Ju, B. et al. Human neutralizing antibodies elicited by SARS-CoV-2 infection. Nature 584, 115-119 (2020).

19. Shi, R. et al. A human neutralizing antibody targets the receptor-binding site of SARS-CoV-2. Nature 584, 120-124 (2020).

20. Wang, Q. et al. Structural and functional basis of SARS-CoV-2 entry by using human ACE2. Cell 181, 894-904 (2020). e899.

21. Letko, M., Marzi, A. \& Munster, V. Functional assessment of cell entry and receptor usage for SARS-CoV-2 and other lineage B betacoronaviruses. Nat. Microbiol. 5, 562-569 (2020).

22. Lv, H. et al. Cross-reactive antibody response between SARS-CoV-2 and SARS-CoV infections. Cell Rep. 31, 107725 (2020).

23. Shrock, E. et al. Viral epitope profiling of COVID-19 patients reveals crossreactivity and correlates of severity. Science 370, eabd4250 (2020).

24. Van Elslande, J. et al. Antibody response against SARS-CoV-2 spike protein and nucleoprotein evaluated by four automated immunoassays and three ELISAs. Clin. Microbiol. Infect. 26, 1557.e1-1557.e7 (2020).

25. Mateus, J. et al. Selective and cross-reactive SARS-CoV-2 T cell epitopes in unexposed humans. Science 370, 89-94 (2020)

26. de Vries, R. D. SARS-CoV-2-specific T-cells in unexposed humans: presence of cross-reactive memory cells does not equal protective immunity. Signal Transduct. Target Ther. 5, 224 (2020).

27. Braun, J. et al. SARS-CoV-2-reactive T cells in healthy donors and patients with COVID-19. Nature 587, 270-274 (2020).

28. Grifoni, A. et al. Targets of T cell responses to SARS-CoV-2 coronavirus in humans with COVID-19 disease and unexposed individuals. Cell 181, 1489-1501 (2020). e1415.

29. Meckiff, B. J. et al. Single-cell transcriptomic analysis of SARS-CoV-2 reactive CD4 (+) T cells. https://doi.org/10.1101/2020.06.12.148916 (2020).

30. Weiskopf, D. et al. Phenotype and kinetics of SARS-CoV-2-specific T cells in COVID-19 patients with acute respiratory distress syndrome. Sci. Immunol. 5, eabd2071 (2020).

31. Che, X. Y. et al. Antigenic cross-reactivity between severe acute respiratory syndrome-associated coronavirus and human coronaviruses 229E and OC43. J. Infect. Dis. 191, 2033-2037 (2005).

32. Nguyen-Contant, P. et al. S protein-reactive IgG and memory B cell production after human SARS-CoV-2 infection includes broad reactivity to the S2 subunit. mBio 11, e01991-20 (2020).

33. Ng, K. W. et al. Preexisting and de novo humoral immunity to SARS-CoV-2 in humans. Science 370, 1339-1343 (2020).

34. Kelvin, A. A. \& Zambon, M. Influenza imprinting in childhood and the influence on vaccine response later in life. Euro Surveill. 24, 1900720 (2019)

35. Fonville, J. M. et al. Antibody landscapes after influenza virus infection or vaccination. Science 346, 996-1000 (2014).
36. Dugan, H. L. et al. Preexisting immunity shapes distinct antibody landscapes after influenza virus infection and vaccination in humans. Sci. Transl. Med. 12, eabd3601 (2020).

37. Erbelding, E. J. et al. A universal influenza vaccine: the strategic plan for the national institute of allergy and infectious diseases. J. Infect. Dis. 218, 347-354 (2018).

38. Monto, A. S., Malosh, R. E., Petrie, J. G. \& Martin, E. T. The doctrine of original antigenic sin: separating good from evil. J. Infect. Dis. 215, 1782-1788 (2017).

39. Gostic, K. M. et al. Childhood immune imprinting to influenza A shapes birth year-specific risk during seasonal H1N1 and H3N2 epidemics. PLoS Pathog. 15, e1008109 (2019).

40. Meade, P. et al. Influenza virus infection induces a narrow antibody response in children but a broad recall response in adults. mBio 11, e03243-19 (2020).

41. Halstead, S. B., Rojanasuphot, S. \& Sangkawibha, N. Original antigenic sin in dengue. Am. J. Trop. Med. Hyg. 32, 154-156 (1983).

42. Midgley, C. M. et al. An in-depth analysis of original antigenic sin in dengue virus infection. J. Virol. 85, 410-421 (2011).

43. Gorse, G. J., Patel, G. B., Vitale, J. N. \& O’Connor, T. Z. Prevalence of antibodies to four human coronaviruses is lower in nasal secretions than in serum. Clin. Vaccin. Immunol. 17, 1875-1880 (2010).

44. Del Valle, D. M. et al. An inflammatory cytokine signature predicts COVID19 severity and survival. Nat. Med. 26, 1636-1643 (2020).

45. Tortorici, M. A. et al. Ultrapotent human antibodies protect against SARSCoV-2 challenge via multiple mechanisms. Science 370, 950-957 (2020).

46. Addetia, A. et al. Neutralizing antibodies correlate with protection from SARS-CoV-2 in humans during a fishery vessel outbreak with high attack rate. J. Clin. Microbiol. 58, e02107-20 (2020).

47. Hassan, A. O. et al. A SARS-CoV-2 infection model in mice demonstrates protection by neutralizing antibodies. Cell 182, 744-753 (2020). e744.

48. Saletti, G. et al. Older adults lack SARS CoV-2 cross-reactive T lymphocytes directed to human coronaviruses OC43 and NL63. Sci. Rep. 10, 21447 (2020).

49. Amanat, F. \& Krammer, F. SARS-CoV-2 vaccines: status report. Immunity 52, 583-589 (2020).

50. Amanat, F. et al. The plasmablast response to SARS-CoV-2 mRNA vaccination is dominated by non-neutralizing antibodies that target both the NTD and the RBD. https://doi.org/10.1101/2021.03.07.21253098 (2021).

51. Aydillo, T. et al. Pre-existing hemagglutinin stalk antibodies correlate with protection of lower respiratory symptoms in flu-infected transplant patients. Cell Rep. Med. 1, 100130 (2020).

52. Amanat, F. et al. A serological assay to detect SARS-CoV-2 seroconversion in humans. Nat. Med. 26, 1033-1036 (2020).

53. Stadlbauer, D. et al. SARS-CoV-2 seroconversion in humans: a detailed protocol for a serological assay, antigen production, and test setup. Curr. Protoc. Microbiol. 57, e100 (2020).

\section{Acknowledgements}

We thank the BACO cohort patients and their families. We also thank Randy Albrecht for support with the BSL3 facility and procedures at the ISMMS and Richard Cadagan for excellent technical assistance. We also want to thank Kizzmekia Corbett and Barney Graham at the Vaccine Research Center at NIAID for sharing expression plasmids for hCoV spike proteins. This work was partly funded by CRIP (Center for Research on Influenza Pathogenesis), a NIAID funded Center of Excellence for Influenza Research and Surveillance (CEIRS, contract \#HHSN272201400008C), by SEM-CIVIC, a NIAID funded Collaborative Influenza Vaccine Innovation Center (contract \#75N93019C00051), by NCI SeroNet grant U54CA260560, and by the generous support of the JPB Foundation, the Open Philanthropy Project (research grant 2020-215611 (5384)) and anonymous donors to A.G.S. A.R. is a recipient of a predoctoral fellowship from the Instituto de Salud Carlos III, Spanish Ministry of Science, Innovation and Universities (PFIS contract FI18/00183).

\section{Author contributions}

T.A. performed experiments, analyzed data, and wrote the manuscript. A.R., G.A.A., and J.C. collected samples and data. D.S., S.A., A.E., K.J., and F.A. performed experiments. F.K. provided reagents, methods, and expertise. T.A. and A.G.S. conceived and designed the study. T.A., J.C., and A.G.S. supervised the study. All of the authors reviewed and edited the manuscript.

\section{Competing interests}

A.G.S. is inventor of patents owned by the Icahn School of Medicine at Mount Sinai in the field of influenza virus vaccines. The A.G.S. lab has received research funds from Avimex, GSK, and 7Hills to investigate novel influenza virus vaccines. The Icahn School of Medicine at Mount Sinai has filed patent applications relating to SARS-CoV-2 serological assays and NDV-based SARS-CoV-2 vaccines which list F.K. as co-inventor. D.S. and F.A. are also listed on the serological assay patent application as co-inventors. Mount Sinai has spun out a company, Kantaro, to market serological tests for SARS-CoV-2. F.K. has consulted for Merck and Pfizer (before 2020), and is currently consulting for Pfizer, Seqirus, and Avimex. The Krammer laboratory is also collaborating with Pfizer on animal models of SARS-CoV-2. The other authors declare no competing interests. 


\section{Additional information}

Supplementary information The online version contains supplementary material available at https://doi.org/10.1038/s41467-021-23977-1.

Correspondence and requests for materials should be addressed to F.K., J.C. or A.G.-S.

Peer review information Nature Communications thanks Galit Alter and Anna Durbin for their contribution to the peer review of this work.

Reprints and permission information is available at http://www.nature.com/reprints

Publisher's note Springer Nature remains neutral with regard to jurisdictional claims in published maps and institutional affiliations. (c) (1) Open Access This article is licensed under a Creative Commons Attribution 4.0 International License, which permits use, sharing, adaptation, distribution and reproduction in any medium or format, as long as you give appropriate credit to the original author(s) and the source, provide a link to the Creative Commons license, and indicate if changes were made. The images or other third party material in this article are included in the article's Creative Commons license, unless indicated otherwise in a credit line to the material. If material is not included in the article's Creative Commons license and your intended use is not permitted by statutory regulation or exceeds the permitted use, you will need to obtain permission directly from the copyright holder. To view a copy of this license, visit http://creativecommons.org/licenses/by/4.0/.

(C) The Author(s) 2021 